FedUni ResearchOnline

http://researchonline.federation.edu.au

This is the author's preprint version of the following publication:

Gao, D. (2016) Analytical solutions to general anti-plane shear problems in finite elasticity. Continuum Mechanics and

Thermodynamics, 28(1-2), 175-194.

The version displayed here may differ from the final published version.

The final publication is available at Springer via:

http://doi.org/10.1007/s00161-015-0412-y

Copyright $@ 2015$, Springer-Verlag Berlin Heidelberg 


\title{
Analytical Solutions to General Anti-Plane Shear Problems In Finite Elasticity
}

\author{
David Yang Gao \\ Federation University Australia, Mt Helen, VIC 3353, Australia \\ Research School of Engineering, Australian National University, Canberra, Australia
}

Dedicated to Professor Ray Ogden on the occasion of his 70th birthday

\begin{abstract}
This paper presents a pure complementary energy variational method for solving anti-plane shear problem in finite elasticity. Based on the canonical duality-triality theory developed by the author, the nonlinear/nonconex partial differential equation for the large deformation problem is converted into an algebraic equation in dual space, which can, in principle, be solved to obtain a complete set of stress solutions. Therefore, a general analytical solution form of the deformation is obtained subjected to a compatibility condition. Applications are illustrated by examples with both convex and nonconvex stored strain energies governed by quadratic-exponential and power-law material models, respectively. Results show that the nonconvex variational problem could have multiple solutions at each material point, the complementary gap function and the triality theory can be used to identify both global and local extremal solutions, while the popular (poly-, quasi-, and rank-one) convexities provide only local minimal criteria, the Legendre-Hadamard condition does not guarantee uniqueness of solutions. This paper demonstrates again that the pure complementary energy principle and the triality theory play important roles in finite deformation theory and nonconvex analysis.
\end{abstract}

AMS Classification: 35Q74, 49S05, 74B20

Keywords: Nonlinear elasticity, Nonlinear PDEs, Canonical duality-triality, Complementary variational principle, Nonconvex analysis.

\section{Introduction}

Anti-plane shear deformation problems arise naturally from many real world applications, such as rectilinear steady flow of simple fluids [10, interface stress effects of nanostructured materials [52], structures with cracks [61], layered/composite functioning materials [56, 171], 
and phase transitions in solids [67]. During the past half century, such problems in finite deformation theory have been subjected to extensively study by both mathematicians and engineering scientists [3, 36, 38, 39, 45, 62, 63]. As indicated in the review article by C.O. Horgan [42]), anti-plane shear deformations are one of the simplest classes of deformations that solids can undergo. In anti-plane shear (or longitudinal shear, generalized shear) of a cylindrical body, the displacement is parallel to the generators of the cylinder and is independent of the axial coordinate. In recent years, considerable attention has been paid to the analysis of anti-plane shear deformations within the context of various constitutive theories (linear and nonlinear) of solid mechanics. Such studies were largely motivated by the promise of relative analytic simplicity compared with plane problems since the governing equations are a single second-order partial differential equation rather than higher-order or coupled systems of partial differential equations. Thus the anti-plane shear problem plays a useful role as a pilot problem, within which various aspects of solutions in solid mechanics may be examined in a particularly simple setting.

Generally speaking, the anti-plane shear problem in linear elasticity is governed by linear partial differential equation, which can be solved easily by well-developed analytical methods. However, in finite elasticity, the associated variational problem is usually nonlinear or nonconvex, which could have multiple solutions. Traditional methods for solving nonconvex variational problems are proved to be very difficult, or even impossible. The well-known generalized convexities and Legendre-Hadamard condition can be used only for identifying local minimal solutions. Numerical methods (such as FEM and FDM, etc) for solving nonconvex variational problems lead to a global optimization problem. Due to the lacking of global optimality condition, most of nonconvex optimization problems are considered to be NP-hard in nonconvex optimization and computer science [30, 55]. Extensive research has been focused on solving such nonconvex optimization problems, and a special research field, i.e., the global optimization has been developed during the past 15 years [29].

Complementary variational principles and methods play important roles in continuum mechanics. It is known that in finite deformation theory, the Hellinger-Reissner principle (see [37, 64]) and the Fraeijs de Veubeke principle (see [69]) hold for both convex and nonconvex problems. But, these well-known principles are not considered as the pure complementary variational principles since the Hellinger-Reissner principle involves both the displacement field and the second Piola-Kirchhoff stress tensor, and the Fraeijs de Veubeke principle needs both the rotation tensor and the first Piola-Kirchhoff stress as its variational arguments. Therefore, the question about the existence of a pure complementary variational principle in general finite deformation theory was argued for several decades (see [47, 48, 49, 60, 57, 58]). A systematic study on the invariant conditions for various complementary energy functionals in elasticity was given by Li and Gupta [51]. Also, since the extremality condition is a fundamentally difficult problem in nonconvex variational analysis and global optimization, all the classical complementary-dual variational principles and associated numerical methods can't be used for solving nonconvex variational/optimization problems in finite deformation theory.

Canonical duality-triality is a newly developed and powerful methodological theory, 
which is composed mainly of a canonical transformation, a pure complementary-dual energy variational principle, and a triality theory. The canonical transformation can be used to model complex systems within a unified framework and to establish perfect dual problems in nonconvex analysis and global optimization. The pure complementary-dual variational principle shows that a class of nonlinear partial differential equations are equivalent to certain algebraic equations which can be solved to obtain analytical solutions in stress space. The triality theory comprises a canonical min-max duality and a pair of doublemin, double-max dualities. The canonical min-max duality can be used to identify global minimizer; while the double-min and double-max dualities can be used to identify local minimizer and local maximizer, respectively. The original idea of the canonical duality theory was introduced by Gao and Strang in general nonconvex variational problems [31. The triality theory was discovered in post-buckling analysis of a large deformed beam model [13]. The pure complementary principle was first proposed in 1999 [15], which has been used successfully for solving finite deformation problems. In a set of papers published recently by Gao and Ogden [27, 26], it is shown that by using this theory, complete sets of analytical solutions can be obtained for one-dimensional nonlinear/nonconvex problems. Their results illustrated an important fact that smooth analytic or numerical solutions of a nonlinear mixed boundary-value problem might not be minimizers of the associated potential variational problem. For global optimization problems in finite dimensional space, the canonical duality theory has been used successfully for and global optimization, see 30]

The purpose of this paper is to illustrate the application of this pure complementary variational principle and the triality theory by solving nonlinear and nonconvex variational problems in anti-plane shear deformation. The remainder article is organized as the following. The next section discusses the finite anti-plane shear deformation and constitutive laws. Based on the equilibrium equation and a general constitutive law, a nonlinear potential variational problem is formulated. Section 3 shows how this nonlinear potential variational problem can be transformed as a canonical dual problem such that a pure complementary energy principle can be obtained, and by which, how the nonlinear partial differential equation for deformation can be converted into an algebraic equation in stress space, so that an analytical solution form for the displacement can be formulated. This section also shows how the global optimal solution can be identified by the triality theory. Section 4 presents an application to convex problem governed by a quadratic-exponential stored energy, which has a unique solution. While for nonconvex strain energy, Section 5 shows that the boundary value problem is not equivalent to the variational problem. By using the canonical dual transformation and the pure complementary variational principle, the nonlinear differential equation can be converted into a cubic algebraic equation, which possesses at most three real roots. Therefore a complete set of solutions to the potential variational problem is obtained. The triality theory can be used to identify global and local minimizers.

The results presented in this paper show that the pure complementary energy principle and the triality theory are potentially useful in finite deformation theory. 


\section{Anti-plane shear deformation and variational prob- lem}

Consider a homogeneous, isotropic elastic cylinder $\mathcal{B} \subset \mathbb{R}^{3}$ with generators parallel to the $\mathbf{e}_{3}$ axis and with cross section a sufficiently nice region $\Omega \subset \mathbb{R}^{2}$ in the $\mathbf{e}_{1} \times \mathbf{e}_{2}$ plane. The so-called anti-plane shear deformation is defined by

$$
\chi=\mathbf{x}+u\left(x_{1}, x_{2}\right) \mathbf{e}_{3}, \quad \forall\left(x_{1}, x_{2}\right) \in \Omega
$$

where $\left(x_{1}, x_{2}, x_{3}\right)$ are cylindrical coordinates in the reference configuration (assumed free of stress) $\mathcal{B}$ relative to a cylindrical basis $\left\{\mathbf{e}_{\alpha}\right\}, \alpha=1,2,3$, and $u: \Omega \rightarrow \mathbb{R}$ is the amount of shear (locally a simple shear) in the planes normal to $\mathbf{e}_{3}$.

We suppose that on boundary $\Gamma_{u} \subset \partial \Omega$ the homogenous boundary condition is given, i.e.,

$$
u(\mathbf{x})=0 \quad \forall \mathbf{x} \in \Gamma_{u},
$$

while on the boundary $\Gamma_{t}=\partial \Omega \cap \Gamma_{u}$, the shear force is prescribed

$$
\mathbf{t}(\mathbf{x})=t(\mathbf{x}) \mathbf{e}_{3} \forall \mathbf{x} \in \Gamma_{t}
$$

The deformation gradient tensor, denoted $\mathbf{F}$, can be readily calculated for the deformation of the form (1):

$$
\mathbf{F}=\nabla \boldsymbol{\chi}=\mathbf{I}+\mathbf{e}_{3} \otimes(\nabla u)=\left(\begin{array}{ccc}
1 & 0 & 0 \\
0 & 1 & 0 \\
u_{, 1} & u_{, 2} & 1
\end{array}\right),
$$

where $u_{, \alpha}$ represents $\partial u / \partial x_{\alpha}$ for $\alpha=1,2, \mathbf{I}$ is the identity tensor; while the corresponding left Cauchy-Green tensor, denoted $\mathbf{B}$, is

$$
\mathbf{B}=\mathbf{F F}^{\mathrm{T}}=\left(\begin{array}{ccc}
1 & 0 & u_{, 1} \\
0 & 1 & u_{, 2} \\
u_{, 1} & u_{, 2} & 1+|\nabla u|^{2}
\end{array}\right),
$$

where the notation ${ }^{\mathrm{T}}$ indicates the transpose (of a second-order tensor).

The principal invariants of $\mathbf{B}$, denoted $I_{1}, I_{2}, I_{3}$, are defined by

$$
I_{1}=\operatorname{tr} \mathbf{B}, \quad I_{2}=\frac{1}{2}\left[(\operatorname{tr} \mathbf{B})^{2}-\operatorname{tr}(\mathbf{B})^{2}\right], \quad I_{3}=\operatorname{det} \mathbf{B},
$$

and, for the considered anti-plane shear problem, these reduce to

$$
I_{1}=I_{2}=3+|\nabla u|^{2}, \quad I_{3}=1 .
$$

In this paper, the notation $|\nabla u|^{2}=u_{, 1}^{2}+u_{, 2}^{2}$ represents the Euclidean norm in $\mathbb{R}^{2}$. 
In view of (6), if we let $\gamma=\nabla u$, we may now introduce a new function, denoted $\hat{W}$, such that the stored-energy density function $W(\mathbf{F})$ can be written as

$$
W(\mathbf{F})=\hat{W}(\gamma)
$$

and the associated in-plane Cauchy stress tensor $\boldsymbol{\tau}$

$$
\boldsymbol{\tau}=\frac{\partial \hat{W}}{\partial \gamma}
$$

is the shear stress.

For the deformation and constitutive law discussed in the foregoing sections the equilibrium equation $\operatorname{div} \boldsymbol{\tau}=0$ (in the absence of body forces) has just one non-trivial components, namely the equilibrium equation

$$
\nabla \cdot \boldsymbol{\tau}=\frac{\partial \tau_{1}}{\partial x_{1}}+\frac{\partial \tau_{2}}{\partial x_{2}}=0
$$

Let the kinetically admissible space be defined by

$$
\mathcal{U}_{a}=\left\{u(\mathbf{x}) \in \mathcal{C}[\bar{\Omega} ; \mathbb{R}] \mid \nabla u \in \mathcal{L}^{2 p}\left[\bar{\Omega} ; \mathbb{R}^{2}\right], \quad u(\mathbf{x})=0 \forall \mathbf{x} \in \Gamma_{u}\right\},
$$

where $\bar{\Omega}=\Omega \cup \partial \Omega$ represents the closure of the set $\Omega, \mathcal{L}^{2 p}$ is the standard notation of Lebesgue integrable space with $p \in \mathbb{R}$, which will be discussed in Section 5 . Then the minimal potential energy principle leads to the following variational problem (primal problem $(\mathcal{P})$ for short) for the determination of the deformation function $u$ :

$$
(\mathcal{P}): \min \left\{\Pi(u)=\int_{\Omega} \hat{W}(\nabla u) \mathrm{d} \Omega-\int_{\Gamma_{t}} t u \mathrm{~d} \Gamma \quad \mid u \in \mathcal{U}_{a}\right\}
$$

where $\min \{*\}$ represents for finding minimum value of the statement in $\{*\}$.

The criticality condition $\delta \Pi(u)=0$ leads to the following mixed boundary value problem:

$$
(B V P):\left\{\begin{array}{l}
\nabla \cdot \frac{\partial \hat{W}(\nabla u)}{\partial(\nabla u)}=0 \quad \forall \mathbf{x} \in \Omega, \\
\mathbf{n} \cdot \frac{\partial \hat{W}(\nabla u)}{\partial(\nabla u)}=t \quad \forall \mathbf{x} \in \Gamma_{t} .
\end{array}\right.
$$

Since the stored energy $\hat{W}(\gamma)$ is a nonlinear function of the shear strain, the $(B V P)$ may possess multiple solutions and each solution represents a stationary point of the total potential energy $\Pi(u)$. Therefore, if $\Pi(u)$ is nonconvex, the variational problem $(\mathcal{P})$ is not equivalent the boundary value problem $(B V P)$. Traditional direct methods for solving the nonlinear boundary value problem are usually very difficult, also how to identify the global minimizer in nonconvex analysis is a fundamentally difficult task. It turns out that in general nonlinear elasticity, even some qualitative questions such as regularity and stability are considered as outstanding open problems [3].

On the other hand, let the statically admissible space to be

$$
\mathcal{T}_{a}=\left\{\boldsymbol{\tau} \in \mathcal{C}\left[\bar{\Omega} ; \mathbb{R}^{2}\right] \mid \nabla \cdot \boldsymbol{\tau}(\mathbf{x})=0 \quad \forall \mathbf{x} \in \Omega, \mathbf{n} \cdot \boldsymbol{\tau}(\mathbf{x})=t \forall \mathbf{x} \in \Gamma_{t}\right\}
$$


The complementary variational problem can be stated as the following:

$$
\min \left\{\Pi^{*}(\boldsymbol{\tau})=\int_{\Omega} \hat{W}^{*}(\boldsymbol{\tau}) \mathrm{d} \Omega \mid \boldsymbol{\tau} \in \mathcal{T}_{a}\right\},
$$

where $\hat{W}^{*}(\boldsymbol{\tau})$ is the so-called complementary energy function (or density), defined by the Legendre transformation:

$$
\hat{W}^{*}(\boldsymbol{\tau})=\{\boldsymbol{\gamma} \cdot \boldsymbol{\tau}-\hat{W}(\boldsymbol{\gamma}) \mid \boldsymbol{\tau}=\nabla \hat{W}(\boldsymbol{\gamma})\}
$$

In finite deformation theory, if the strain energy density $\hat{W}(\boldsymbol{\gamma})$ is nonconvex, the Legendre conjugate $\hat{W}^{*}(\boldsymbol{\tau})$ can not be uniquely obtained [59, 65]. In this case, the classical complementary energy variational principle can not be used for solving nonconvex finite deformation problems. Although by the Fenchel transformation

$$
\hat{W}^{\sharp}(\boldsymbol{\tau})=\sup \left\{\boldsymbol{\gamma} \cdot \boldsymbol{\tau}-\hat{W}(\boldsymbol{\gamma}) \mid \boldsymbol{\gamma} \in \mathcal{L}^{2 p}\left[\bar{\Omega} ; \mathbb{R}^{2}\right]\right\},
$$

the Fenchel conjugate $\hat{W}^{\sharp}(\boldsymbol{\tau})$ is always convex, and the Fenchel-Moreau dual problem can be obtained as

$$
\max \left\{\Pi^{\sharp}=-\int_{\Omega} \hat{W}^{\sharp}(\boldsymbol{\tau}) \mathrm{d} \Omega \mid \boldsymbol{\tau} \in \mathcal{T}_{a}\right\},
$$

the Fenchel-Young inequality

$$
\hat{W}(\gamma) \geq \boldsymbol{\gamma} \cdot \boldsymbol{\tau}-\hat{W}^{\sharp}(\boldsymbol{\tau})
$$

leads to

$$
\theta=\min _{u \in \mathcal{U}_{a}} \Pi(u)-\max _{\boldsymbol{\tau} \in \mathcal{T}_{a}} \Pi^{\sharp}(\boldsymbol{\tau}) \geq 0
$$

and the non zero $\theta \neq 0$ is the well-known duality gap in nonconvex analysis. According to Sir M. Atiyah [2], duality in mathematics is not a theorem, but a "principle". Therefore, the duality gap is not allowed in mathematical physics. It turns out that the existence of a pure complementary energy principle in finite elasticity was a well-known open problem which has been discussed for over thirty years. This problem was solved in 1999 [15] when a complementary energy principle was proposed in terms of the first and second Piola-Kirchhoff stresses only.

In the following sections, we will demonstrate the application of the canonical duality theory and the pure complementary variational principle for solving the proposed variational problem. In order to examine this problem in detail, we will consider the energy function in both convex and nonconvex forms.

\section{Canonical dual problem and extremality theory}

The key step of the canonical dual transformation is to introduce a new geometrical measure $\xi=\Lambda(u)$ and a canonical function $V(\xi)$ such that the stored energy function $\hat{W}(\nabla u)=$ 
$V(\Lambda(u))$. By the definition introduced in [17] that a real-valued function $V(\xi)$ is called a canonical function if the duality relation

$$
\zeta=\frac{\partial V(\xi)}{\partial \xi}
$$

is invertible such that the conjugate function $V^{*}(\zeta)$ of $V(\zeta)$ can be defined uniquely by the Legendre transformation:

$$
V^{*}(\zeta)=\left\{\xi \zeta-V(\xi) \mid \zeta=\frac{\partial V(\xi)}{\partial \xi}\right\}
$$

The canonical dual transformation has a solid foundation in physics. According to the frame-invariance axiom [6, 40, 59], instead of the linear deformation $\gamma=\nabla u$, the strain energy $\hat{W}(\gamma)$ should be a function of a quadratic measure $\xi=\Lambda(u)$. In view of (6) and (7), for this anti-plane shear deformation problem we can simply choose the geometrical measure $\xi=\Lambda(u)=\frac{1}{2}|\nabla u|^{2}$, which is a quadratic mapping from $\mathcal{U}_{a}$ to a closed convex set

$$
\mathcal{E}_{a}=\left\{\xi \in \mathcal{L}^{p}[\bar{\Omega} ; \mathbb{R}] \mid \xi(\mathbf{x}) \geq 0 \quad \forall \mathbf{x} \in \Omega\right\}
$$

Let $\mathcal{E}_{a}^{*}$ be the range of the canonical duality mapping $\nabla V: \mathcal{E}_{a} \rightarrow \mathcal{E}^{*}$, such that on $\mathcal{E}_{a} \times \mathcal{E}_{a}^{*}$, the following canonical duality relations hold:

$$
\zeta=\nabla V(\xi) \Leftrightarrow \xi=\nabla V^{*}(\zeta) \Leftrightarrow V(\xi)+V^{*}(\zeta)=\xi \zeta .
$$

In the terminology of finite elasticity, if the geometrical measure $\xi$ can be viewed as a Cauchy-Green type strain, its conjugate $\zeta$ is a second Piola-Kirchhoff type stress. For many hyper-elastic materials, the stored energy function could be nonconvex in the deformation gradient, but is usually convex in the Cauchy-Green type strain measure. Thus, replacing $\hat{W}(\nabla u)$ in the total potential energy $\Pi(u)$ by the canonical form $V(\Lambda(u))$, the primal problem $(\mathcal{P})$ can be written in the following canonical form:

$$
(\mathcal{P}): \min \left\{\Pi(u)=\int_{\Omega} V(\Lambda(u)) \mathrm{d} \Omega-\int_{\Gamma_{t}} u t \mathrm{~d} \Gamma \mid u \in \mathcal{U}_{a}\right\}
$$

Furthermore, by using the Fenchel-Young equality $V(\Lambda(u))=\Lambda(u) \zeta-V^{*}(\zeta)$, the so-called total complementary energy functional originally proposed by Gao and Strang in [31] can be written as

$$
\Xi(u, \zeta)=\int_{\Omega}\left[\frac{1}{2}|\nabla u|^{2} \zeta-V^{*}(\zeta)\right] \mathrm{d} \Omega-\int_{\Gamma_{t}} u t \mathrm{~d} \Gamma .
$$

This two-field functional is well-defined on $\mathcal{U}_{a} \times \mathcal{E}_{a}^{*}$. Let

$$
\mathcal{S}^{+}=\left\{\zeta \in \mathcal{E}_{a}^{*} \mid \zeta(\mathbf{x}) \geq 0 \quad \forall \mathbf{x} \in \Omega\right\} .
$$

The following theorem is a special case of the general result by Gao and Strang [31]. 
Theorem 1 (Complementary-Dual Variational Extremum Principle) If $(\bar{u}, \bar{\zeta})$ is a critical point of $\Xi(u, \zeta)$, then $\bar{u}$ is a local solution to $(B V P)$. Moreover, if $V(\xi)$ is convex and $\bar{\zeta} \in \mathcal{S}^{+}$, then $\bar{u}$ is a global optimal solution to the minimal variational problem $(\mathcal{P})$ and

$$
\Pi(\bar{u})=\min _{u \in \mathcal{U}_{a}} \Pi(u)=\Xi(\bar{u}, \bar{\zeta})=\min _{u \in \mathcal{U}_{a}} \max _{\zeta \in \mathcal{S}^{+}} \Xi(u, \zeta)=\max _{\zeta \in \mathcal{S}^{+}} \min _{u \in \mathcal{U}_{a}} \Xi(u, \zeta) .
$$

Proof. By the criticality condition $\delta \Xi(\bar{u}, \bar{\zeta})=0$, we obtain

$$
\begin{gathered}
\Lambda(\bar{u})=\frac{1}{2}|\nabla \bar{u}|^{2}=\nabla V^{*}(\bar{\zeta}), \quad \text { in } \Omega \\
\nabla \cdot(\bar{\zeta} \nabla \bar{u})=0 \quad \text { in } \Omega, \mathbf{n} \cdot(\bar{\zeta} \nabla \bar{u})=t \text { on } \Gamma_{t} .
\end{gathered}
$$

By the canonical duality (22), we know that the Euler-Lagrangian equation (27) is equivalent to the canonical constitutive-geometrical equation $\bar{\zeta}=\nabla V(\Lambda(\bar{u}))$. Combining this with the equilibrium equation (28) ary value problem $(B V P)$ and $\bar{u}$ is a critical point of the the total potential energy $\Pi(u)$.

By the convexity of the canonical energy $V(\xi)$, we have (see [31])

$$
V(\xi)-V(\bar{\xi}) \geq(\xi-\bar{\xi}) \nabla V(\bar{\xi}) \forall \xi, \bar{\xi} \in \mathcal{E}_{a} .
$$

Let $\xi=\Lambda(u), \bar{\xi}=\Lambda(\bar{u})$, and $\bar{\zeta}=\nabla V(\Lambda(\bar{u}))$, we obtained

$$
\Pi(u)-\Pi(\bar{u}) \geq \int_{\Omega}[\bar{\zeta}(\Lambda(u)-\Lambda(\bar{u}))] \mathrm{d} \Omega-\int_{\Gamma_{t}} t(u-\bar{u}) \mathrm{d} \Gamma \quad \forall u \in \mathcal{U}_{a} .
$$

Let $u=\bar{u}+\delta u$. By the fact that $\Lambda(u)$ is a quadratic operator, we have (see [31])

$$
\Lambda(u)=\Lambda(\bar{u}+\delta u)=\Lambda(\bar{u})+(\nabla \delta u)^{T}(\nabla \bar{u})+\Lambda(\delta u) .
$$

Therefore, if $(\bar{u}, \bar{\zeta})$ is a critical point of $\Xi(u, \zeta)$ and $\bar{\zeta} \in \mathcal{S}^{+}$, we have

$$
\Pi(u)-\Pi(\bar{u})=G(\delta u, \bar{\zeta})=\int_{\Omega} \bar{\zeta} \Lambda(\delta u) \mathrm{d} \Omega \geq 0 \quad \forall \delta u .
$$

This shows that $\bar{u}$ is a global minimizer of $\Pi(u)$ over $\mathcal{U}_{a}$.

\section{Remark 1 (Gao-Strang's Gap Function and Global Optimality Condition)}

Theorem 1 is actually the direct application of the general result of [31], and

$$
G(u, \zeta)=\int_{\Omega} \zeta \Lambda(u) \mathrm{d} \Omega
$$

is the so-called complementary gap function first introduced by Gao and Strang in 1989 [31]. Since the geometrical operator $\Lambda(u)=\frac{1}{2}|\nabla u|^{2}$ is quadratic, the gap function

$$
G(u, \zeta) \geq 0 \forall u \in \mathcal{U}_{a} \text { if and only if } \zeta \in \mathcal{S}^{+}
$$


Therefore, the total complementary energy $\Xi(u, \zeta)$ is a saddle functional on $\mathcal{U}_{a} \times \mathcal{S}^{+}$, i.e.

$$
\Xi(u, \bar{\zeta}) \geq \Xi(\bar{u}, \bar{\zeta}) \geq \Xi(\bar{u}, \zeta) \forall(u, \zeta) \in \mathcal{U}_{a} \times \mathcal{S}^{+}
$$

Thus, by the canonical min-max duality, we have (26). Theorem 1 shows that the gap function $G(u, \bar{\zeta}) \geq 0$ provides a global optimality condition for the nonconvex variational problem $(\mathcal{P})$. This gap function also plays a key role in global optimization (see [30]). Based on this complementary extremum principle and the general canonical primal-dual mixed finite element method [5, 35], an efficient algorithm can be developed for solving general anti-plane shear problems.

By the virtual work principle, for any given statically admissible $\boldsymbol{\tau} \in \mathcal{T}_{a}$, we have

$$
\int_{\Omega}(\nabla u) \cdot \boldsymbol{\tau} \mathrm{d} \Omega=\int_{\Gamma}(\boldsymbol{\tau} \cdot \mathbf{n}) u \mathrm{~d} \Gamma-\int_{\Omega}(\nabla \cdot \boldsymbol{\tau}) u \mathrm{~d} \Omega=\int_{\Gamma_{t}} t u \mathrm{~d} \Gamma \quad \forall u \in \mathcal{U}_{a} .
$$

Replacing the boundary integral in (24) by (29), the total complementary energy functional $\Xi(u, \zeta)$ can be written as

$$
\Xi_{\boldsymbol{\tau}}(u, \zeta)=\int_{\Omega}\left[\frac{1}{2}|\nabla u|^{2} \zeta-V^{*}(\zeta)-(\nabla u) \cdot \boldsymbol{\tau}\right] \mathrm{d} \Omega .
$$

Theorem 2 For any given statically admissible $\boldsymbol{\tau} \in \mathcal{T}_{a}$, if $(\bar{u}, \bar{\zeta})$ is a critical point of $\Xi_{\boldsymbol{\tau}}(u, \zeta)$, then it is also a critical point of $\Xi(u, \zeta)$, and

$$
\Pi(\bar{u})=\Xi(\bar{u}, \bar{\zeta})=\Xi_{\boldsymbol{\tau}}(\bar{u}, \bar{\zeta}) \quad \forall \boldsymbol{\tau} \in \mathcal{T}_{a} .
$$

Proof. For a given $\boldsymbol{\tau} \in \mathcal{T}_{a}$, the criticality condition $\delta \Xi \boldsymbol{\tau}(\bar{u}, \bar{\zeta})=0$ gives to the inverse constitutive law $\frac{1}{2}|\nabla \bar{u}|^{2}=\nabla V^{*}(\bar{\zeta})$ and the balance equations

$$
\nabla \cdot(\bar{\zeta} \nabla \bar{u})=\nabla \cdot \boldsymbol{\tau} \text { in } \Omega, \mathbf{n} \cdot(\bar{\zeta} \nabla \bar{u})=\mathbf{n} \cdot \boldsymbol{\tau} \quad \text { on } \Gamma_{t} .
$$

Therefore, $(\bar{u}, \bar{\zeta})$ is a critical point of $\Xi(u, \zeta)$, and also a solution to $(B V P)$ for any given $\tau \in \mathcal{T}_{a}$. The equality (31) can be proved easily by the virtual work principle and the canonical duality relations (22).

This theorem shows that the statically admissible stress field $\tau \in \mathcal{T}_{a}$ does not change the value of the functional $\Xi_{\boldsymbol{\tau}}(u, \zeta)$. Note that from the criticality conditions (32), we have

$$
\zeta \nabla u=\tau,
$$

which shows the relation between the canonical stress and the first Piola-Kirchhoff stress. By substituting $\nabla u=\boldsymbol{\tau} / \zeta$ in $\Xi_{\boldsymbol{\tau}}(u, \zeta)$, the pure complementary energy $\Pi^{d}(\zeta)$ can be obtained by the canonical dual transformation [16]

$$
\Pi^{d}(\zeta)=\operatorname{sta}\left\{\Xi_{\boldsymbol{\tau}}(u, \zeta) \mid \forall u \in \mathcal{U}_{a}\right\}=-\int_{\Omega}\left(\frac{|\boldsymbol{\tau}|^{2}}{2 \zeta}+V^{*}(\zeta)\right) \mathrm{d} \Omega
$$


which is well-defined on

$$
\mathcal{S}_{a}^{+}=\left\{\left.\zeta \in \mathcal{S}^{+}|| \boldsymbol{\tau}\right|^{2} / \zeta \in \mathcal{L}[\bar{\Omega} ; \mathbb{R}]\right\}
$$

Therefore, the complementary variational problem which is canonically dual to the potential variational problem $(\mathcal{P})$ can be proposed as

$$
\left(\mathcal{P}^{d}\right): \max \left\{\Pi^{d}(\zeta)=-\int_{\Omega}\left(\frac{|\boldsymbol{\tau}|^{2}}{2 \zeta}+V^{*}(\zeta)\right) \mathrm{d} \Omega \mid \zeta \in \mathcal{S}_{a}^{+}\right\}
$$

According to [17], we have the following result.

Theorem 3 (Pure Complementary Energy Principle) For a given statically admissible $\boldsymbol{\tau} \in \mathcal{T}_{a}$, if $(\bar{u}, \bar{\zeta})$ is a critical point of $\Xi_{\boldsymbol{\tau}}(u, \zeta)$, then $\bar{\zeta}$ is a critical point of $\Pi^{d}(\zeta), \bar{u}$ is a critical point of $\Pi(u)$, and

$$
\Pi(\bar{u})=\Xi_{\boldsymbol{\tau}}(\bar{u}, \bar{\zeta})=\Pi^{d}(\bar{\zeta})
$$

If $V(\xi)$ is convex, then $\bar{u}$ is a global minimum solution to $(\mathcal{P})$ if and only if $\bar{\zeta} \in \mathcal{S}_{a}^{+}$is a solution to $\left(\mathcal{P}^{d}\right)$, i.e.

$$
\Pi(\bar{u})=\min _{u \in \mathcal{U}_{a}} \Pi(u) \Leftrightarrow \max _{\zeta \in \mathcal{S}_{a}^{+}} \Pi^{d}(\zeta)=\Pi^{d}(\bar{\zeta})
$$

The problem $(\mathcal{P})$ has a unique solution if $\bar{\zeta}(\mathbf{x})>0, \forall \mathbf{x} \in \Omega$.

Proof. By the equation (31) we know that for a given $\boldsymbol{\tau} \in \mathcal{T}_{a}$, the functionals $\Xi(u, \zeta)$ and $\Xi_{\boldsymbol{\tau}}(u, \zeta)$ have the same critical points set. Particularly, the criticality condition condition $\delta \Pi^{d}(\bar{\zeta})=0$ leads to

$$
\frac{|\boldsymbol{\tau}|^{2}}{2(\bar{\zeta})^{2}}=\nabla V^{*}(\bar{\zeta})
$$

which is in fact the inverse constitutive-geometrical equation (27) subject to

$$
\nabla \bar{u}=\frac{\tau}{\bar{\zeta}}
$$

Since $\tau$ is statically admissible, therefore, $\bar{\zeta} \nabla \bar{u}=\boldsymbol{\tau}$ satisfies equilibrium conditions (28). This proved that the critical point $\bar{\zeta}$ of the canonical dual problem $\left(\mathcal{P}^{d}\right)$ and the associated $\bar{u}$ are also critical point of $\Xi$.

Again by the canonical duality (22), we have

$$
\Xi(\bar{u}, \bar{\zeta})=\int_{\Omega} V(\Lambda(\bar{u})) \mathrm{d} \Omega-\int_{\Gamma_{t}} t \bar{u} \mathrm{~d} \Gamma=\int_{\Omega} \hat{W}(\nabla \bar{u}) \mathrm{d} \Omega-\int_{\Gamma_{t}} t \bar{u} \mathrm{~d} \Gamma=\Pi(\bar{u}) .
$$

Dually, by using (39), we have for any given $\boldsymbol{\tau} \in \mathcal{T}_{a}$

$$
\Xi(\bar{u}, \bar{\zeta})=\Xi \boldsymbol{\tau}(\bar{u}, \bar{\zeta})=\Pi^{d}(\bar{\zeta})
$$


By the fact that $\Xi_{\boldsymbol{\tau}}(u, \zeta)$ is a saddle functional on $\mathcal{U}_{a} \times \mathcal{S}_{a}^{+}$, we have

$$
\min _{u \in \mathcal{U}_{a}} \Pi(u)=\min _{u \in \mathcal{U}_{a}} \max _{\zeta \in \mathcal{S}_{a}^{+}} \Xi \boldsymbol{\tau}(u, \zeta)=\max _{\zeta \in \mathcal{S}_{a}^{+}} \min _{u \in \mathcal{U}_{a}} \Xi_{\boldsymbol{\tau}}(u, \zeta)=\max _{\zeta \in \mathcal{S}_{a}^{+}} \Pi^{d}(\zeta) .
$$

Thus, $\bar{u} \in \mathcal{U}_{a}$ is a global minimum solution to $(\mathcal{P})$ if and only if $\bar{\zeta} \in \mathcal{S}_{a}^{+}$is a solution to the canonical dual problem $\left(\mathcal{P}^{d}\right)$. Moreover, if $\bar{\zeta}(\mathbf{x})>0 \forall \mathbf{x} \in \Omega$, then the gap function $G(u, \bar{\zeta})>0 \forall u \neq 0$. From the proof of Theorem 1 we know that the total potential energy $\Pi(u)$ is strictly convex on $\mathcal{U}_{a}$, therefore, the global min is unique.

Remark 2 This theorem shows that the complementary energy variational problem $\left(\mathcal{P}^{d}\right)$ is canonically dual to the potential variational problem $(\mathcal{P})$, i.e. there is no duality gap. The canonical dual Euler-Lagrangian equation (38) shows that the criticality condition of the pure complementary energy is an algebraic equation

$$
\bar{\zeta}^{2} \nabla V^{*}(\bar{\zeta})=\frac{1}{2}|\boldsymbol{\tau}|^{2}
$$

which can be solved easily for many real applications. Therefore, the pure complementary energy principle plays an important role in stress analysis and design. But, for each $\tau$, the solution $\bar{\zeta}$ can only produce the deformation gradient $\nabla \bar{u}=\bar{\zeta}^{-1} \boldsymbol{\tau}$. In order to obtain the primal solution $\bar{u}$ by solving the canonical dual problem, additional compatibility condition is needed.

Theorem 4 (Analytical Solution Form) For a given statically admissible stress $\boldsymbol{\tau}(\mathbf{x}) \in$ $\mathcal{T}_{a}$ such that $\bar{\zeta}(\mathbf{x})$ is a solution of the canonical dual equation 40), then the vector-valued function

$$
\nabla \bar{u}=\bar{\zeta}^{-1}(\mathbf{x}) \boldsymbol{\tau}
$$

is a deformation solution to the $(B V P)$.

Moreover, if $\boldsymbol{\tau} \in \mathcal{T}_{a}$ is a potential field and

$$
\boldsymbol{\tau} \times(\nabla \bar{\zeta})=0 \quad \forall \mathbf{x} \in \Omega
$$

then the path-independent line integral

$$
\bar{u}(\mathbf{x})=\int_{\mathbf{x}_{0}}^{\mathbf{x}} \bar{\zeta}^{-1} \boldsymbol{\tau} \cdot \mathrm{d} \mathbf{x} \quad \forall \mathbf{x}_{0} \in \Gamma_{u}
$$

is a solution of the boundary value problem $(B V P)$.

Proof. First, by using chain role we know that if $\nabla \bar{u}$ is a solution to $(B V P)$, it must satisfy

$$
\frac{\partial \hat{W}(\nabla \bar{u})}{\partial(\nabla \bar{u})}=\frac{\partial V(\Lambda(\bar{u}))}{\partial \Lambda(\bar{u})} \frac{\partial \Lambda(\bar{u})}{\partial \nabla \bar{u}}=\bar{\zeta} \nabla \bar{u} \in \mathcal{T}_{a} .
$$


Let $\boldsymbol{\tau}=\bar{\zeta} \nabla \bar{u} \in \mathcal{T}_{a}$, we have

$$
\nabla \bar{u}=\bar{\zeta}^{-1} \boldsymbol{\tau}
$$

which is indeed the critical condition $\delta_{u} \Xi \boldsymbol{\tau}(\bar{u}, \bar{\zeta})=0$. By the canonical duality (22), we have

$$
\frac{1}{2}|\nabla \bar{u}|^{2}=\frac{1}{2} \frac{|\boldsymbol{\tau}|^{2}}{\bar{\zeta}^{2}}=\nabla V^{*}(\bar{\zeta})
$$

which is the canonical dual algebraic equation, i.e., the criticality condition of $\delta \Pi^{d}(\bar{\zeta})=0$. Therefore, for a given $\tau \in \mathcal{T}_{a}$, if $\bar{\zeta}$ is a solution to this canonical dual equation (40), then $\nabla \bar{u}=\bar{\zeta}^{-1} \boldsymbol{\tau}$ is the deformation field of the $(B V P)$.

Moreover, if $\bar{u}$ can be solved by the path-independent line integral (43), the integrant $\bar{\zeta}^{-1} \boldsymbol{\tau}$ must be a potential field on $\Omega$, i.e. $\nabla \times\left(\bar{\zeta}^{-1} \boldsymbol{\tau}\right)=0$. This leads to

$$
\bar{\zeta}(\nabla \times \boldsymbol{\tau})+\boldsymbol{\tau} \times(\nabla \bar{\zeta})=0 \quad \text { on } \Omega .
$$

Since $\boldsymbol{\tau}(\mathbf{x})$ is a potential field on $\Omega$, i.e., there exists a scale-valued function $\phi(\mathbf{x})$ such that $\boldsymbol{\tau}=\nabla \phi(\mathbf{x})$, then we have $\nabla \times \boldsymbol{\tau}=\nabla \times(\nabla \phi) \equiv 0$ on $\Omega$. Therefore, as long as the condition $\boldsymbol{\tau} \times(\nabla \bar{\zeta})=0$ holds on $\Omega$, the deformation gradient $\nabla \bar{u}=\bar{\zeta}^{-1} \boldsymbol{\tau}$ is a potential field and the displacement $\bar{u}$ can be obtained by the path-integral (43). By the fact that $\bar{u}\left(\mathbf{x}_{0}\right)=0$, it should be an analytical solution to $(B V P)$.

Generally speaking, the canonical dual algebraic equation (40) is nonlinear which allows multiple solutions for nonconvex problems. In the following sections, we shall present some applications.

\section{Application to Convex Variational Problem}

First, we assume the stored energy $\hat{W}(\boldsymbol{\gamma})$ is a convex function of the type (see [26]):

$$
\hat{W}(\boldsymbol{\gamma})=\frac{1}{2} \mu|\gamma|^{2}+\nu\left(\exp \left(\frac{1}{2}|\boldsymbol{\gamma}|^{2}\right)-1\right)
$$

where $\mu>0$ and $\nu>0$ are material constants. In this case, the constitutive equation (8) can be written as the following form

$$
\boldsymbol{\tau}(\boldsymbol{\gamma})=\nabla \hat{W}(\boldsymbol{\gamma})=\mu \boldsymbol{\gamma}+\nu \boldsymbol{\gamma} \exp \left(\frac{1}{2}|\boldsymbol{\gamma}|^{2}\right)
$$

which can be used to model a large class of materials, especially bio-materials (cf. [41]). The associated potential variational problem is

$$
\left(\mathcal{P}_{1}\right): \min _{u \in \mathcal{U}_{a}}\left\{\Pi(u)=\int_{\Omega}\left[\frac{1}{2} \mu|\nabla u|^{2}+\nu\left(\exp \left(\frac{1}{2}|\nabla u|^{2}\right)-1\right)\right] \mathrm{d} \Omega-\int_{\Gamma_{t}} t u \mathrm{~d} \Gamma\right\} .
$$

This problem also appears in the construction of optimal Lipschitz extensions of given boundary data, the Monge - Kantorovich optimal mass transfer problem, and a form of 
weak KAM theory for Hamiltonian dynamics (see [4]), etc. Although the energy function is convex and the constitutive relation is monotone (see Fig. 1), the complementary energy $\hat{W}^{*}(\boldsymbol{\tau})$ can not be obtained by the Legendre transformation since the inverse relation of $\boldsymbol{\tau}(\boldsymbol{\gamma})$ is analytically impossible.


Figure 1: Graphs of $\hat{W}(\gamma)$ (left) and its derivative (right) $(\mu=1.0, \nu=0.5)$

By using the geometrical measure $\xi=\Lambda(u)=\frac{1}{2}|\nabla u|^{2}$, the canonical energy function can be defined by

$$
V(\xi)=\mu \xi+\nu(\exp (\xi)-1),
$$

and $\hat{W}(\boldsymbol{\gamma})=V(\xi(\boldsymbol{\gamma}))$. Clearly, the canonical strain energy $V(\xi)$ is well-defined on the domain $\mathcal{E}_{a}$. Thus, the constitutive law (46) can be written in the following simple form:

$$
\zeta=V^{\prime}(\xi)=\mu+\nu \exp (\xi)
$$

which is uniquely defined on the domain

$$
\mathcal{E}_{a}^{*}=\{\zeta \in \mathcal{C}[\Omega ; \mathbb{R}] \mid \zeta(\mathbf{x}) \geq \mu+\nu \forall \mathbf{x} \in \Omega\} .
$$

Therefore, the complementary energy $V^{*}(\zeta): \mathcal{E}_{a}^{*} \rightarrow \mathbb{R}$ can be obtained easily as

$$
V^{*}(\zeta)=\operatorname{sta}\left\{\xi \zeta-V(\xi) \mid \xi \in \mathcal{E}_{a}\right\}=(\zeta-\mu)\left(\log \left(\frac{\zeta-\mu}{\nu}\right)-1\right)+\nu .
$$

Clearly, the canonical duality relations (22) hold on $\mathcal{E}_{a} \times \mathcal{E}_{a}^{*}$.

By the fact that on $\mathcal{E}_{a}^{*}$, we have $\mathcal{S}_{a}=\mathcal{E}_{a}^{*}=\mathcal{S}_{a}^{+}$and the canonical stress $\zeta(\mathbf{x}) \geq \mu+\nu>$ $0 \forall \mathbf{x} \in \Omega$, the total complementary energy $\Xi(u, \zeta)$ (or $\left.\Xi_{\boldsymbol{\tau}}(u, \zeta)\right)$ is convex in $u \in \mathcal{U}_{a}$ and the pure complementary variational problem $\left(\mathcal{P}^{d}\right)$ for this convex problem can be written in the following

$$
\left(\mathcal{P}_{1}^{d}\right): \max _{\zeta \in \mathcal{S}_{a}}\left\{\Pi_{1}^{d}(\zeta)=-\int_{\Omega}\left(\frac{|\boldsymbol{\tau}|^{2}}{2 \zeta}+(\zeta-\mu)\left(\log \left(\frac{\zeta-\mu}{\nu}\right)-1\right)+\nu\right) \mathrm{d} \Omega\right\} .
$$


Theorem 5 For a given statically admissible stress $\boldsymbol{\tau} \in \mathcal{T}_{a}$, the canonical dual problem $\left(\mathcal{P}_{1}^{d}\right)$ has a unique solution $\bar{\zeta}(\mathbf{x}) \geq \mu+\nu$. If $\boldsymbol{\tau}$ is a potential field and $\boldsymbol{\tau} \times(\nabla \bar{\zeta})=0$ on $\Omega$, then the function

$$
\bar{u}(\mathbf{x})=\int_{\mathbf{x}_{0}}^{\mathbf{x}}(\bar{\zeta}(\mathbf{x}))^{-1} \boldsymbol{\tau} \cdot \mathrm{d} \mathbf{x} \quad \forall \mathbf{x}_{0} \in \Gamma_{u}
$$

is a unique solution of $\left(\mathcal{P}_{1}\right)$ and

$$
\Pi_{1}(\bar{u})=\min _{u \in \mathcal{U}_{a}} \Pi_{1}(u)=\max _{\zeta \in \mathcal{S}_{a}} \Pi_{1}^{d}(\zeta)=\Pi_{1}^{d}(\bar{\zeta}) .
$$

Proof. The criticality condition $\delta \Pi_{1}^{d}(\zeta)=0$ leads to the dual algebraic equation

$$
2 \zeta^{2} \log \left(\frac{\zeta-\mu}{\nu}\right)=|\boldsymbol{\tau}|^{2}
$$

Let $h^{2}(\zeta)=2 \zeta^{2} \log \left(\frac{\zeta-\mu}{\nu}\right)$. Then the graph of $h(\zeta)= \pm \zeta \sqrt{2 \log ((\zeta-\mu) / \nu)}$ is the so-called dual algebraic curve (see Fig. 2). Clearly, for any given $|\boldsymbol{\tau}|$, the canonical dual algebraic equation (55) has a unique solution $\bar{\zeta} \in \mathcal{S}_{a}$. Since the canonical complementary energy

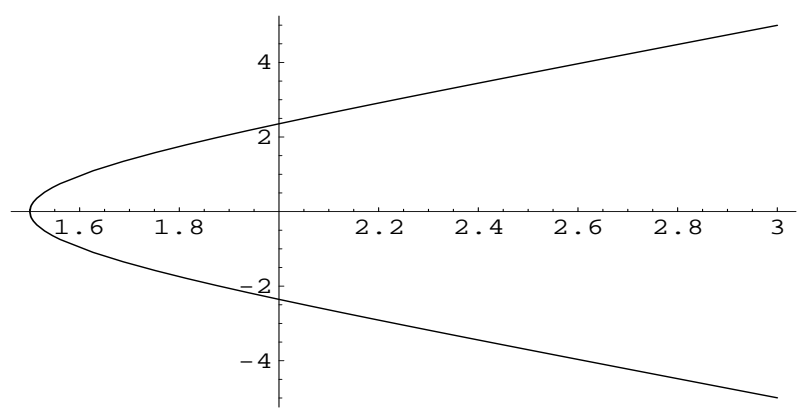

Figure 2: Dual algebraic curve $h(\zeta)(\mu=1, \quad \nu=0.5)$

density

$$
\psi(\zeta)=\frac{|\boldsymbol{\tau}|^{2}}{2 \zeta}+(\zeta-\mu)\left(\log \left(\frac{\zeta-\mu}{\nu}\right)-1\right)+\nu
$$

is a strictly convex function of $\zeta$ on the dual feasible space $\mathcal{S}_{a}$, for a given shear stress $\boldsymbol{\tau}$, the pure complementary energy $\Pi_{1}^{d}(\zeta)$ is strictly concave on $\mathcal{S}_{a}=\mathcal{S}_{a}^{+}$. Therefore, the solution $\bar{\zeta}$ of the canonical dual algebraic equation (55) should a unique global maximizer $\bar{\zeta} \geq \mu+\nu$. By Theorem 4 we know that if the condition $\tau \times(\nabla \bar{\zeta})=0$, the analytic solution $\bar{u}$ can be determined by (43). 


\section{Application to Nonconvex Power-Law Material Model}

In this section the stored strain energy is assume to be a polynomial function of the shear strain $|\gamma|$ :

$$
\hat{W}(\gamma)=\frac{\mu}{2 b}\left[\left(1+\frac{b}{p}\left(|\gamma|^{2}-\epsilon\right)\right)^{p}-1\right]
$$

where $\mu>0$ is the infinitesimal shear modulus, $p, b>0$ are material parameters, and $\epsilon \in \mathbb{R}$ is a given (internal) parameter, which can be viewed as, for examples, residue strain [16], dislocation [22], random defects [24], or input control in functioning materials (see [28]). If $\epsilon=0$, this is the power-law material model introduced by Knowles in 1977 [46], and in this case, the energy function possesses the following properties:

$$
\hat{W}(0)=0, \quad \nabla \hat{W}(0)=0, \quad \nabla^{2} \hat{W}(0)=\mu \mathbf{I} \succ 0,
$$

which are necessary for $\hat{W}(\boldsymbol{\gamma})$ to be a stored energy. The associated stress in simple shear is

$$
\boldsymbol{\tau}=\mu\left(1+\frac{b}{p}\left(|\gamma|^{2}-\epsilon\right)\right)^{p-1} \boldsymbol{\gamma}
$$

The power-law material hardens or softens in shear according to whether $p>1$ or $p<1$. Graphs of this material model are shown in Figure 3. Particularly, when $p=\frac{1}{2}, \epsilon=0$, the partial differential equation $\nabla \cdot \nabla \hat{W}(\nabla u)=0$ becomes

$$
\left(1+2 b u_{, 2}^{2}\right) u_{, 11}-4 b u_{, 1} u_{, 2} u_{, 12}+\left(1+2 b u_{, 1}^{2}\right) u_{, 22}=0,
$$

which, on re-scaling $u$ (or by letting $2 b=1$ ), is the celebrated minimal surface equation

$$
\left(1+u_{, 2}^{2}\right) u_{, 11}-2 u_{, 1} u_{, 2} u_{, 12}+\left(1+u_{, 1}^{2}\right) u_{, 22}=0 .
$$

It also governs the flow of a Kármán-Tsien gas (see [42]).

It is easy to prove that for $p \geq \frac{1}{2}$, the stored energy $\hat{W}(\gamma)$ is convex (see Section 6.5.3, [17]). In this case, the $(B V P)$ is elliptic (see [46]). However, if $p<\frac{1}{2}$, the constitutive law $\boldsymbol{\tau}=\nabla \hat{W}(\boldsymbol{\gamma})$ is not monotone even if $\epsilon=0$ (see Fig. 3 (a)). Although it can be considered for modeling softening phenomenon, this case is not physically allowed since $p<\frac{1}{2}$ violates the constitutive law. Mathematical explanation for this case can be given by the canonical duality theory (see below).

When $p=1$ in (56), the stored energy function $\hat{W}(\gamma)$ is linear, which recovers the neo-Hookean material.

For $p>1$, the stored energy function $\hat{W}(\gamma)$ is convex if $\epsilon \leq 0$, nonconvex for $\epsilon>0$. Particularly, if $p=2$ and $\epsilon>p / b$, this nonconvex function $\hat{W}(\gamma)$ is the so-called doublewell energy in mathematical physics (see Fig. 3 (b)), which appears frequently in phase transitions of solids, Landau-Ginzburg model in super-conductivity [22], post-buckling of large deformed beam [5], as well as in quantum mechanics such as Higgs mechanism and Yang-Mills fields etc. For $p>2$, the stored energy and constitutive law are shown in Fig. 3 (c). 

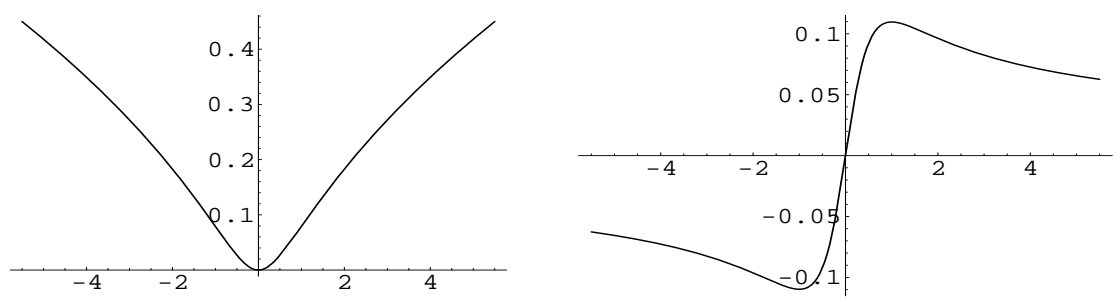

(a) $p=0.25, \epsilon=0$.
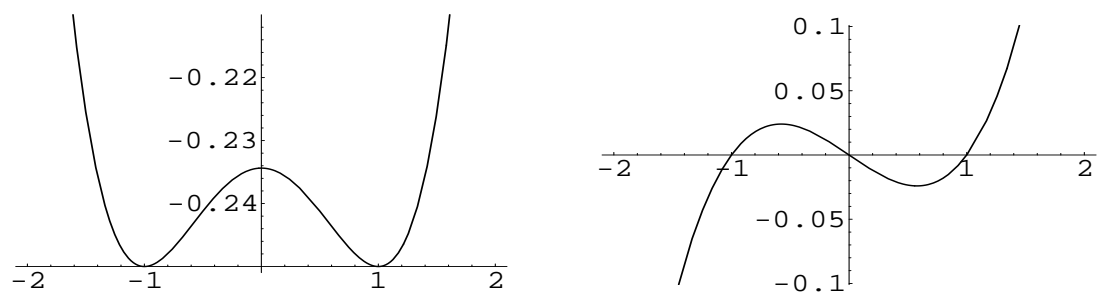

(b) $p=2, \quad \epsilon=2.5$.
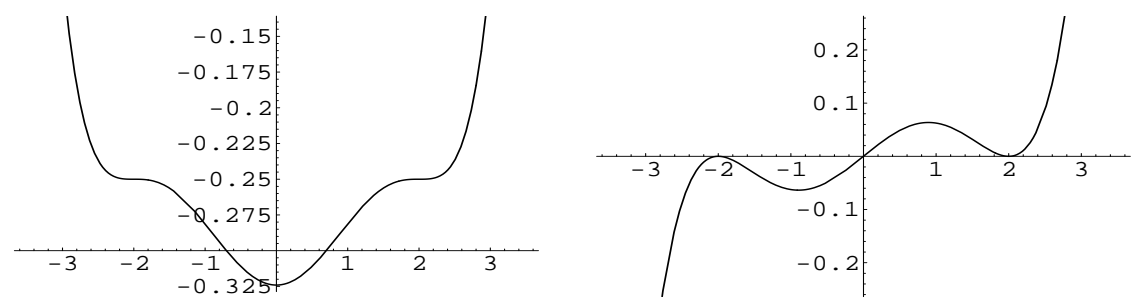

(c) $p=3, \quad \epsilon=5$.

Figure 3: Nonconvex energy $\hat{W}(\gamma)$ (left) and non-monotone constitutive law $\hat{W}^{\prime}(\gamma)$ (right), $\mu=0.5, b=1$.

Let $\beta=\mu b^{p-1} / p^{p}, \quad \alpha=\epsilon-p / b$, and $\beta_{o}=\mu /(2 b)$. The minimal potential energy principle leads to the following nonlinear variational extremum problem:

$$
\left(\mathcal{P}_{2}\right): \min \left\{\Pi_{2}(u)=\int_{\Omega}\left[\frac{1}{2} \beta\left(|\nabla u|^{2}-\alpha\right)^{p}-\beta_{o}\right] \mathrm{d} \Omega-\int_{\Gamma_{t}} t u \mathrm{~d} \Gamma \mid u \in \mathcal{U}_{a}\right\} .
$$

For certain given parameter $\alpha>0$, this variational problem is nonconvex and the corresponding boundary-value problem $(B V P)$ is not equivalent to $\left(\mathcal{P}_{2}\right)$ since the solution of $(B V P)$ may not be the global minimizer of $\left(\mathcal{P}_{2}\right)$. Due to the lack of global optimality condition, traditional direct methods for solving the $\left(\mathcal{P}_{2}\right)$ are very difficult.

By using the canonical strain measure $\Lambda(u)=|\nabla u|^{2}$, the canonical function for this power-law model can be defined by

$$
V(\xi)=\frac{1}{2} \beta(\xi-\alpha)^{p}-\beta_{o}
$$

which is defined on the closed convex domain

$$
\mathcal{E}_{a}=\left\{\xi \in \mathcal{L}^{p} \mid \xi(\mathbf{x}) \geq 0, \quad \forall \mathbf{x} \in \Omega\right\} .
$$


Clearly, this canonical function is convex for $p \geq 1$, but nonconvex for $p<1$. In any case, the canonical dual stress is uniquely obtained by

$$
\zeta=\nabla V(\xi)=\frac{1}{2} p \beta(\xi-\alpha)^{p-1},
$$

which is well defined on the dual space

$$
\mathcal{E}_{a}^{*}=\left\{\zeta \in \mathcal{L}^{p /(p-1)} \mid \zeta(\mathbf{x}) \geq \frac{1}{2} \mu(-\alpha b / p)^{p-1} \forall \mathbf{x} \in \Omega\right\} .
$$

Thus the complementary energy can be simply obtained by the traditional Legendre transformation

$$
V^{*}(\zeta)=\{\xi \cdot \zeta-V(\xi) \mid \zeta=\nabla V(\xi)\}=\frac{p-1}{p} c \zeta^{p /(p-1)}+\alpha \zeta+\beta_{o}
$$

where $c=\left(\frac{2}{p \beta}\right)^{1 /(p-1)}=\left(\frac{2}{\mu}\right)^{1 /(p-1)} p / b$. The corresponding total complementary energy for this nonconvex problem is

$$
\Xi(u, \zeta)=\int_{\Omega}\left[\left(|\nabla u|^{2}-\alpha\right) \zeta-\frac{p-1}{p} c \zeta^{p /(p-1)}-\beta_{o}\right] \mathrm{d} \Omega-\int_{\Gamma_{t}} t u \mathrm{~d} \Gamma .
$$

Therefore, for a given $\tau \in \mathcal{T}_{a}$, let

$$
\mathcal{S}_{a}^{+}=\left\{\left.\zeta \in \mathcal{E}_{a}^{*}|\zeta(\mathbf{x}) \geq 0 \forall \mathbf{x} \in \Omega,| \boldsymbol{\tau}\right|^{2} / \zeta \in \mathcal{L}\right\} .
$$

The canonical dual problem in this nonconvex case is

$$
\left(\mathcal{P}_{2}^{d}\right): \max \left\{\Pi_{2}^{d}(\zeta)=-\int_{\Omega}\left[\frac{|\boldsymbol{\tau}|^{2}}{4 \zeta}+\frac{p-1}{p} c \zeta^{p /(p-1)}+\alpha \zeta+\beta_{o}\right] \mathrm{d} \Omega \mid \zeta \in \mathcal{S}_{a}\right\}
$$

The criticality condition $\delta \Pi_{2}^{d}(\zeta)=0$ leads to the dual algebraic equation:

$$
4 \zeta^{2}\left(c \zeta^{1 /(p-1)}+\alpha\right)=|\tau|^{2} .
$$

The solutions of this algebraic equation depends mainly on the material parameter $p>0$. It can be easily checked by using MATHEMATICA that if $p<\frac{1}{2}$, this equation has no real root. For $p=\frac{1}{2}$, the equation (65) has real roots only under the condition $|\boldsymbol{\tau}|^{2} \leq \frac{\mu^{2}}{2 b}$. Particularly, for minimal surface-type problems where $\mu=1$ and $2 b=1$, the condition $|\boldsymbol{\tau}|^{2} \leq 1$ verifies the result presented in [17] (Section 6.5.3). Canonical duality theory for solving minimal surface type problems have been studied in [34]. In this paper, we are interested in $p>1$ with positive internal parameter $\alpha>0$ such that the stored energy is nonconvex, which can be used to model more interesting phenomena.

Particularly, for $p=2$, the canonical dual algebraic (65) is cubic

$$
4 \zeta^{2}(c \zeta+\alpha)=|\boldsymbol{\tau}|^{2}
$$


which can be solved analytically to have three solutions:

$$
\begin{aligned}
& \bar{\zeta}_{1}=-\frac{\alpha}{3 c}+\frac{2^{4 / 3} \alpha^{2}}{3 c \psi(\tau)}+\frac{\psi(\tau)}{3(2)^{4 / 3} c} \\
& \bar{\zeta}_{2}=-\frac{\alpha}{3 c}-\frac{2^{1 / 3} \alpha^{2}(1-i \sqrt{3})}{3 c \psi(\tau)}-\frac{(1+i \sqrt{3}) \psi(\tau)}{12\left(2^{1 / 3}\right) c}, \\
& \bar{\zeta}_{3}=-\frac{\alpha}{3 c}-\frac{2^{1 / 3} \alpha^{2}(1+i \sqrt{3})}{3 c \psi(\tau)}-\frac{(1-i \sqrt{3}) \psi(\tau)}{12\left(2^{1 / 3}\right) c}
\end{aligned}
$$

where $\tau=|\tau|$, and

$$
\psi(\tau)=\left(-16 \alpha^{3}+27 c^{2} \tau^{2}+3 \sqrt{3} \tau \sqrt{-32 \alpha^{3} c^{2}+27 c^{4} \tau^{2}}\right)^{1 / 3} .
$$

Similar to the general results proposed in [14, 18, 26], we have the following theorems.

Theorem 6 (Criteria for Multiple Solutions ) For a given parameter $\alpha \in \mathbb{R}$ and the material constants $p=2, \mu, b>0$ such that $c=4 /(\mu b)>0$, let

$$
\eta=\frac{16 \alpha^{3}}{27 c^{2}}
$$

If $\eta \leq 0$, the $(B V P)$ has a unique solution in the whole domain $\Omega$.

If $\eta>0$, then the $(B V P)$ could have multi-solutions in $\Omega$. In this case, if $\boldsymbol{\tau}$ is a given shear stress and

$$
|\boldsymbol{\tau}(\mathbf{x})|^{2}>\eta \forall \mathbf{x} \in \Omega,
$$

the dual algebraic equation (66) has a unique real root $\bar{\zeta}(\mathbf{x})>0$. If $|\boldsymbol{\tau}|^{2}<\eta$, the dual algebraic equation (65) has three real roots in the order of

$$
\bar{\zeta}_{1}(\mathbf{x}) \geq 0 \geq \bar{\zeta}_{2}(\mathbf{x}) \geq \bar{\zeta}_{3}(\mathbf{x}) .
$$

Proof. Similar to the proof of the Corollary 1 in [18], we let $h^{2}(\zeta)=4 \zeta^{2}(c \zeta+\alpha)$ be the left hand side function in the dual algebraic equation (66). By solving $h^{\prime}\left(\zeta_{c}\right)=0$ we known that $h(\zeta)$ has a local maximum $h_{\max }^{2}\left(\zeta_{c}\right)=\eta$ at $\zeta_{c}=-\frac{2 \alpha}{3 c}$. From the graphs of the dual algebraic curve $h(\zeta)= \pm 2 \zeta \sqrt{c \zeta+\alpha}$ given in Fig. 伎 we can see that if $\eta<0$, the dual algebraic equation (66) has a unique solution for any given $\boldsymbol{\tau}$. However, if $\eta>0$, the dual algebraic equation (66) may have at most three real solutions in the order of (70)

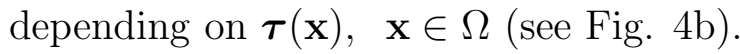

Theorem 7 (Global \& Local Extrema, Uniqueness, and Smoothness) Suppose for a given external force $t(\mathbf{x})$ on $\Gamma_{t}$ that $\boldsymbol{\tau} \in \mathcal{T}_{a}$ is a statically admissible shear force field. If $\boldsymbol{\tau}(\mathbf{x})$ is not identical zero over the domain $\Omega$, the canonical dual problem $\left(\mathcal{P}_{2}^{d}\right)$ has at most three solutions $\bar{\zeta}_{i}(\mathbf{x})(i=1,2,3)$ defined analytically by (67,69), and

$$
\bar{u}_{i}=\int_{\mathbf{x}_{0}}^{\mathbf{x}}\left(2 \bar{\zeta}_{i}(\mathbf{x})\right)^{-1} \boldsymbol{\tau} \cdot d \mathbf{x}, \quad i=1,2,3
$$




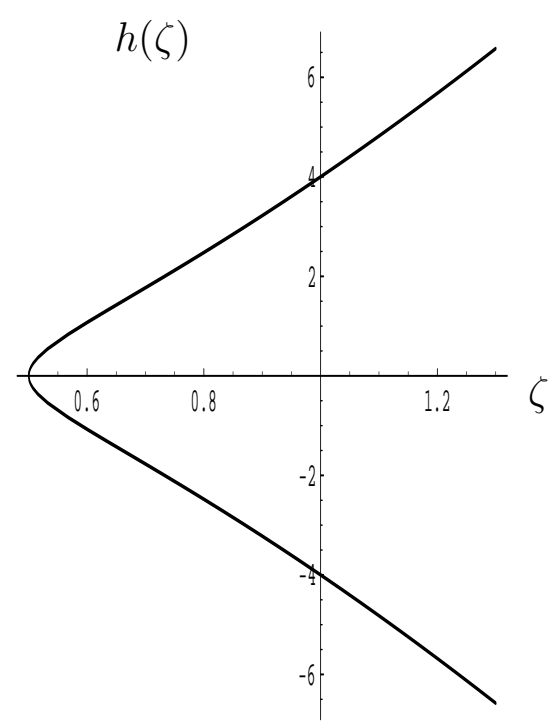

(a) $\eta<0(\alpha=-4)$.

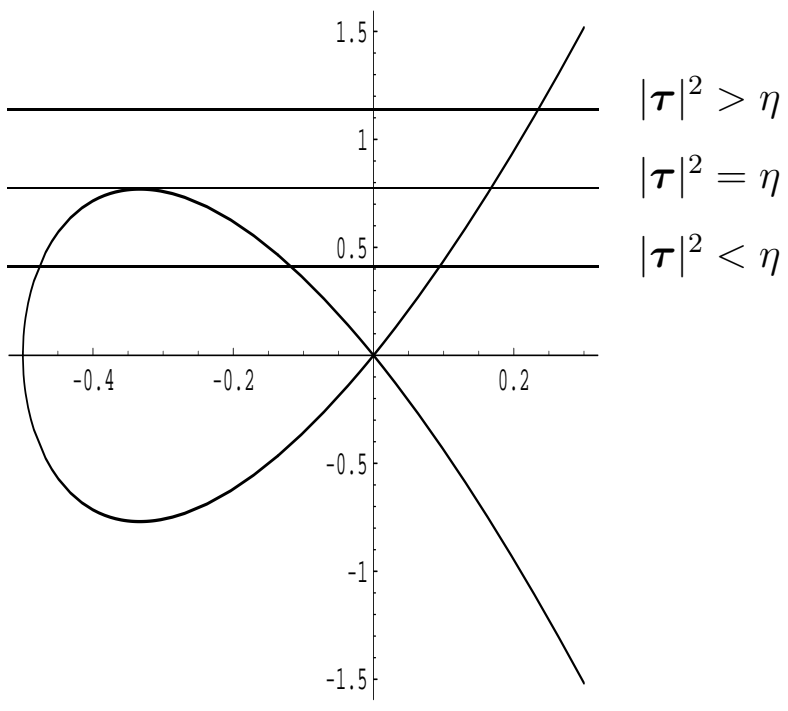

(b) $\eta>0(\alpha=4)$

Figure 4: Dual algebraic curve $h(\zeta)= \pm 2 \zeta \sqrt{c \zeta+\alpha}(\mu=1, \quad b=0.5)$

are the critical solutions to $\left(\mathcal{P}_{2}\right)$. Particularly, $\bar{\zeta}_{1}(\mathbf{x})$ is a global maximizer of $\Pi_{2}^{d}$ over $\mathcal{S}_{a}^{+}$, the associated $\bar{u}_{1}$ is a global minimizer of $\Pi_{2}$ on $\mathcal{U}_{a}$, and

$$
\Pi_{2}\left(\bar{u}_{1}\right)=\min _{u \in \mathcal{U}_{a}} \Pi_{2}(u)=\max _{\zeta \in \mathcal{S}_{a}^{+}} \Pi_{2}^{d}(\zeta)=\Pi_{2}^{d}\left(\bar{\zeta}_{1}\right) .
$$

If $|\boldsymbol{\tau}(\mathbf{x})|^{2}<\eta \forall \mathbf{x} \in \Omega$, then $\bar{\zeta}_{3}(\mathbf{x})$ and the associated $\bar{u}_{3}$ are local maximizers of $\Pi_{2}^{d}$ and $\Pi_{2}$, respectively, and

$$
\Pi_{2}\left(\bar{u}_{3}\right)=\max _{u \in \mathcal{U}_{3}} \Pi_{2}(u)=\max _{-\alpha \beta<\zeta<-2 \alpha \beta / 3} \Pi_{2}^{d}(\zeta)=\Pi_{2}^{d}\left(\bar{\zeta}_{3}\right) ;
$$

If $|\boldsymbol{\tau}(\mathbf{x})|^{2}<\eta \quad \forall \mathbf{x} \in \Omega \subset \mathbb{R}$, then $\bar{\zeta}_{2}(\mathbf{x})$ and the associated $\bar{u}_{2}$ are local minimizers of $\Pi_{2}^{d}$ and $\Pi_{2}$, respectively, and

$$
\Pi_{2}\left(\bar{u}_{2}\right)=\min _{u \in \mathcal{U}_{2}} \Pi_{2}(u)=\min _{-2 \alpha \beta / 3<\zeta<0} \Pi_{2}^{d}(\zeta)=\Pi_{2}^{d}\left(\bar{\zeta}_{2}\right),
$$

where $\mathcal{U}_{2}$ and $\mathcal{U}_{3}$ are neighborhoods of $\bar{u}_{2}$ and $\bar{u}_{3}$, respectively.

If $|\boldsymbol{\tau}(\mathbf{x})|^{2}>\eta>0 \forall \mathbf{x} \in \Omega$, the canonical dual problem $\left(\mathcal{P}_{2}^{d}\right)$ has a unique solution $\bar{\zeta}_{1}(\mathbf{x})$ over $\Omega$ and the primal solution $\bar{u}_{1}$ is a unique smooth global minimizer of $\Pi_{2}(u)$ over $\mathcal{U}_{a}$.

Proof. If $\zeta \in \mathcal{S}_{a}^{+}$, the total complementary energy $\Xi(\xi, \zeta)$ is a saddle functional. The proof of the canonical min-max duality (72) follows directly from Gao and Strang's work [31] and the canonical duality theory [17]. If $\zeta<0$, the total complementary energy $\Xi(\xi, \zeta)$ is concave in both $\xi$ and $\zeta$. The double-max duality (73) is simply due to the fact

$$
\max _{u \in \mathcal{U}_{a}} \Pi_{2}(u)=\max _{u} \max _{\zeta} \Xi_{\boldsymbol{\tau}}(u, \zeta)=\max _{\zeta} \max _{u} \Xi_{\boldsymbol{\tau}}(u, \zeta)=\max _{\zeta} \Pi_{2}^{d}(\zeta)
$$


Note that the double-min duality (74) holds only for $\Omega \subset \mathbb{R}$. Therefore, by considering $\nabla u=u^{\prime}=\gamma$ and the canonical transformation $\hat{W}(\gamma)=V(\xi(\gamma))$, we have

$$
\nabla^{2} \hat{W}(\gamma)=2 \nabla V(\xi)+4 \gamma^{2} \nabla^{2} V(\xi)=2 \zeta+\beta(\tau / \zeta)^{2}
$$

which is positive for any $\zeta>0$. Therefore, $u_{1}$ is a global minimizer of $\Pi_{2}$. By Theorem 6, it is easy to verify that

$$
\nabla^{2} \hat{W}(\gamma) \begin{cases}>0 & \text { if } \zeta \in(-2 \alpha \beta / 3,0) \\ =0 & \text { if } \tau^{2}=\eta, \zeta=\zeta_{c}=-2 \alpha \beta / 3 \\ <0 & \text { if } \zeta \in(-\alpha \beta,-2 \alpha \beta / 3)\end{cases}
$$

This shows that $\hat{W}(\gamma)$ is locally convex at $\gamma_{2}=\tau /\left(2 \zeta_{2}\right)$ and concave at $\gamma_{3}=\tau /\left(2 \zeta_{3}\right)$. Therefore $u_{2}$ is a local minimizer, while $u_{3}$ is a local maximizer of $\Pi(u)$.

By the fact that $\tau(\mathbf{x})=|\boldsymbol{\tau}(\mathbf{x})| \geq 0 \quad \forall \mathbf{x} \in \Omega$, the force field $\tau(\mathbf{x})$ does not cross the Maxwell line, i.e. the $\zeta$-axis in Fig. 4 (see Theorem 1 in [27]). Therefore, by Theorem 6 in [27], the global optimal solution should be smooth over the whole domain $\Omega$.

Remark 3 (Triality Theory \& Ellipticity Condition) By Theorems 6 and 7 we know that if $|\boldsymbol{\tau}(\mathbf{x})|^{2}<\eta \forall \mathbf{x} \in \Omega$, nonconvex problem $\left(\mathcal{P}_{2}\right)$ has three sets of solutions $\left\{\bar{u}_{i}(\mathbf{x})\right\}(i=$ $1,2,3)$ at each $\mathbf{x} \in \Omega$ defined by (71). The global minimizer $\bar{u}_{1}$ is identified by the canonical min-max duality (72), which was first proposed by Gao and Strang in 1989 [31]. The (biggest) local maximizer $\bar{u}_{3}$ is identified by the double-max duality (73). Although the canonical dual solution $\bar{\zeta}_{2}$ is a local minimizer of $\Pi_{2}^{d}$, the associated $\bar{u}_{2}$ is a local minimizer of $\Pi_{2}(u)$ governed by the double-min duality (74) only if the domain $\Omega$ is a subset of $\mathbb{R}$. The triality theory was originally proposed and proved for one-dimension problems $\Omega \subset \mathbb{R}$ by Gao in 1996-2000 [13, 17, 18]. However, in 2003 some counterexamples were discovered which show that the double-min duality holds under certain additional constraints (see Remark 1 in [20] and Remark in [21], page 288). This open problem has been solved in 2010 first in global optimization, i.e. the "certain additional constraints" are simply that the primal and dual problems should have the same dimension in order to have strong triality theory. Otherwise, the double-min duality holds weakly in a subspace [32, 33].

Ellipticity condition has been emphasized to play a fundamental role for the existence of solutions in nonlinear elasticity [10]. However, this is only for convex problems. From Theorem 7 we know that a nonconvex finite deformation problem could have multiple critical solutions at each material point and the associated Euler equation may not be elliptic at all. Therefore, the triality theory reveals an important fact in nonconvex analysis and nonlinear elasticity, i.e. the Legendre-Hadamard condition does not guarantee uniqueness of solutions and the equilibrium equation may not be elliptic even if the L.H. holds at certain local solutions. 


\section{Objectivity, Canonical Duality, and Gap Function}

The main goal of this section is to discuss some concepts in canonical duality theory and their important roles in finite elasticity and nonconvex analysis. Standard notations in 3-D nonlinear elasticity are adopted.

For a general finite deformation problem $\chi: \Omega \subset \mathbb{R}^{3} \rightarrow \omega \subset \mathbb{R}^{3}$, the stored energy function $W(\mathbf{F})$ is usually a nonconvex function of the deformation gradient $\mathbf{F}=\nabla \boldsymbol{\chi} \in$ $\mathbb{M}_{+}^{3}=\left\{\mathbf{F}=\left\{F_{\alpha}^{i}\right\} \in \mathbb{R}^{3 \times 3} \mid \operatorname{det} F>0\right\}$. Thus, the boundary-value problem

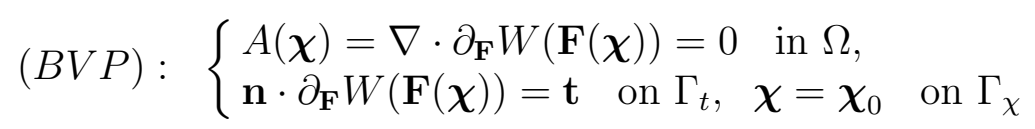

may have multiple solutions at each material point $\mathrm{x} \in \Omega$. According to Dacorogna [8, the following statements, essentially due to Morrey [54], are well-known:

(I) $W(\mathbf{F})$ is convex $\Rightarrow$ poly-convex $\Rightarrow$ quasi-convex $\Rightarrow$ rank-one convex.

(II) If $\Omega \subset \mathbb{R}$ or $\omega \subset \mathbb{R}$, all these notions are equivalent.

(III) If $W \in \mathcal{C}^{2}\left(\mathbb{M}_{+}^{3}\right)$, then the rank-one convexity is equivalent to the LegendreHadamard (L.H.) condition:

$$
\sum_{i, j=1}^{3} \sum_{\alpha, \beta=1}^{3} \frac{\partial^{2} W(\mathbf{F})}{\partial F_{\alpha}^{i} \partial F_{\beta}^{j}} a_{i} a_{j} b^{\alpha} b^{\beta} \geq 0 \quad \forall \mathbf{a}=\left\{a_{i}\right\} \in \mathbb{R}^{3}, \forall \mathbf{b}=\left\{b^{\alpha}\right\} \in \mathbb{R}^{3} .
$$

The Legendre-Hadamard condition in finite elasticity is also referred to as the ellipticity condition, i.e., if the L.H. condition holds, the partial differential operator $A(\boldsymbol{\chi})$ in (77) is considered to be elliptic.

However, all these generalized convexities provide only necessary conditions for local minimal solutions. From the triality theory we know that the nonconvex variational problem may have multiple solutions at each material point $\mathbf{x} \in \Omega$. The conditions in (76) show that even if the Legendre-Hadamard condition holds at the solutions $u_{1}(\mathbf{x})$ and $u_{2}(\mathbf{x})$, the stored energy $\hat{W}(\nabla u)$ is not convex at $\mathbf{x}$ and the differential operator $A(u)$ is not monotone, i.e. the statement (II) is not true! Also, the definition of elliptic operators was originally introduced for linear partial differential equations that generalize the Laplace equation, where, the stored energy is a convex quadratic function and its level set is an ellipse. This definition was generalized for nonlinear operators [9, 66]. From the following discussion, we can see that the stored energy $W(\mathbf{F})$ is not convex even if the L.H. condition holds.

By the fact that the deformation gradient $\mathbf{F}$ is a two-point tensor field, which is not considered as a strain measure. According to the axiom of objectivity or frame-invariance, the following theorem lays a foundation for the canonical duality theory.

Theorem 8 (Theorem 4.2-1 in [7]) The stored energy function of a hyper-elastic material is objective if and only if

$$
W(\mathbf{F})=W(\mathbf{Q F}) \quad \forall \mathbf{F} \in \mathbb{M}_{+}^{3}, \quad \forall \mathbf{Q} \in \mathbb{O}_{+}^{3},
$$


or equivalently, if and only there exists a function $V: \mathbb{S}_{>}^{3} \rightarrow \mathbb{R}$ such that

$$
W(\mathbf{F})=V\left(\mathbf{F}^{T} \mathbf{F}\right) \quad \forall \mathbf{F} \in \mathbb{M}_{+}^{3},
$$

where $\mathbb{O}_{+}^{3}=\left\{\mathbf{Q} \in \mathbb{R}^{3 \times 3} \mid \mathbf{Q}^{-1}=\mathbf{Q}^{T}\right.$, $\left.\operatorname{det} \mathbf{Q}=1\right\}$ is an orthogonal group and $\mathbb{S}_{>}^{3}=\{\mathbf{C} \in$ $\left.\mathbb{R}^{3 \times 3} \mid \mathbf{C}=\mathbf{C}^{T}, \mathbf{C} \succ 0\right\}$.

The objectivity is a fundamental concept in continuum physics (see [17, 40, 53, 59])1 1 . It was emphasized by P.G. Ciarlet that the objectivity is an axiom, not an assumption [6, 7]. According to the traditional philosophical principle of ying-yang duality [12, the constitutive relations in any physical system should be one-to-one in order to obey the fundamental law of nature, i.e. the Dao (I-Ching, 2800-2737 BCE). This one-to-one constitutive relation is called the canonical duality. Therefore, for a given material, it is reasonable to assume the existence of an objective strain measure $\boldsymbol{\xi}=\Lambda(\boldsymbol{\chi}) \in \mathcal{E} \subset \mathbb{S}_{>}^{3}$ and a convex function $V: \mathcal{E} \rightarrow \mathbb{R}$ such that $W(\mathbf{F})=V(\boldsymbol{\xi})$ and the following canonical duality relations hold

$$
\boldsymbol{\xi}^{*}=\nabla V(\boldsymbol{\xi}) \Leftrightarrow \boldsymbol{\xi}=\nabla V^{*}\left(\boldsymbol{\xi}^{*}\right) \Leftrightarrow V(\boldsymbol{\xi})+V^{*}\left(\boldsymbol{\xi}^{*}\right)=\boldsymbol{\xi}: \boldsymbol{\xi}^{*} .
$$

By the canonical transformation $W(\nabla \chi)=V(\Lambda(\chi))$, the general minimal potential problem in finite deformation theory can be written in the following canonical form

$$
\min \left\{\Pi(\chi)=\int_{\Omega} V(\Lambda(\chi)) \mathrm{d} \Omega+F(\chi) \mid \chi \in \mathcal{X}_{a}\right\}
$$

which is the mathematical model studied by Gao and Strang in [31] for general geometrically nonlinear systems, where $F(\chi)$ is the so-called external energy, which should be a linear functional of $\chi$ such that its Gâteaux derivative obeys the Newton third law of action and reaction; the feasible space is defined by

$$
\mathcal{X}_{a}=\left\{\chi \in \mathcal{C}\left[\bar{\Omega} ; \mathbb{R}^{3}\right] \mid \nabla \chi \in \mathbb{M}_{+}^{3}, \quad \chi=\chi_{0} \text { on } \Gamma_{\chi}\right\} .
$$

Canonical duality theory has been extensively studied for different objective measures $\xi=\Lambda(\chi)$ in continuum mechanics and general complex systems [17, 21, 29]. In order to understand why the complementary gap function can be used to identify both global and local extrema, let us consider the most simple canonical strain measure $\boldsymbol{\xi}=\frac{1}{2} \mathbf{F}^{T} \mathbf{F}$ such that $\mathbf{E}=\boldsymbol{\xi}-\frac{1}{2} \mathbf{I}=\frac{1}{2}\left(\mathbf{F}^{T} \mathbf{F}-\mathbf{I}\right)$ is the well-known Green-St Venant strain strain. Its canonical dual is the second Piola-Kirchhoff stress, denoted by $\mathbf{T}=\nabla V(\mathbf{E})$. For a given statically admissible stress $\boldsymbol{\tau} \in \mathcal{T}_{a}=\left\{\boldsymbol{\tau} \in \mathbb{R}^{3 \times 3} \mid \nabla \cdot \boldsymbol{\tau}(\mathbf{x})=0 \quad \forall \mathbf{x} \in \Omega, \quad \mathbf{n} \cdot \boldsymbol{\tau}(\mathbf{x})=\mathbf{t} \forall \mathbf{x} \in \Gamma_{t}\right\}$, the pure complementary energy can be formulated as

$$
\Pi^{d}(\mathbf{T})=\int_{\Gamma_{\chi}} \mathbf{n} \cdot \boldsymbol{\tau} \cdot \chi_{0} \mathrm{~d} \Gamma-\int_{\Omega} \frac{1}{2} \operatorname{tr}\left(\boldsymbol{\tau} \cdot \mathbf{T}^{-1} \cdot \boldsymbol{\tau}+\mathbf{T}\right) \mathrm{d} \Omega-\int_{\Omega} V^{*}(\mathbf{T}) \mathrm{d} \Omega .
$$

\footnotetext{
${ }^{1}$ The concept of objectivity has been misused in mathematical optimization (but mainly in English literature). It turns out that Gao-Strang's work and the canonical duality-triality theory have been challenged recently by C. Zălinescu and his co-workers R. Strugariu, M. D. Voisei (see [68, 70] and references cited therein). Unfortunately, they oppositely chose linear functions as the stored energy $W(\mathbf{F})$ and nonlinear functions as the external energy $F(\chi)$, they produced many interesting "counterexamples" with opposite conclusions. Interested readers are recommended to read 33 for further discussion.
} 
The criticality condition $\delta \Pi^{d}(\mathbf{T})=0$ leads to the canonical dual algebraic equation [15]

$$
\mathbf{T} \cdot\left(2 \nabla V^{*}(\mathbf{T})+\mathbf{I}\right) \cdot \mathbf{T}=\boldsymbol{\tau}^{T} \boldsymbol{\tau} .
$$

Clearly, for a given statically admissible stress field $\boldsymbol{\tau}(\mathbf{x}) \in \mathcal{T}_{a}$, this nonlinear tensor equation may have multiple solutions $\left\{\mathbf{T}_{k}\right\}$, and for each of these critical solutions, the deformation defined by

$$
\chi_{k}(\mathbf{x})=\int_{\mathbf{x}_{0}}^{\mathbf{x}} \tau \mathbf{T}_{k}^{-1} \mathrm{~d} \mathbf{x}+\chi_{0}\left(\mathbf{x}_{0}\right)
$$

along any path from $\mathbf{x}_{0} \in \Gamma_{\chi}$ to $\mathbf{x} \in \Omega$ is a critical point of $\Pi(\boldsymbol{\chi})$ [17]. The vector-valued function $\chi_{k}(\mathbf{x})$ is a solution to the boundary-value problem $(B V P)$ if the compatibility condition $\nabla \times\left(\boldsymbol{\tau} \cdot \mathbf{T}_{k}^{-1}\right)=0$ holds [15]. By Gao-Strang's work [31], $\boldsymbol{\chi}_{k}(\mathbf{x})$ is a global minimizer of $\Pi(\chi)$ if the complementary gap function

$$
G\left(\boldsymbol{\chi}, \mathbf{T}_{k}\right)=\int_{\Omega} \frac{1}{2} \operatorname{tr}\left[(\nabla \boldsymbol{\chi})^{T} \cdot \mathbf{T}_{k} \cdot(\nabla \boldsymbol{\chi})\right] \mathrm{d} \Omega \geq 0 \quad \forall \boldsymbol{\chi} \in \mathcal{X}_{a}
$$

Since this gap function is quadratic in $\chi$, the sufficient condition (88) holds if $\mathbf{T}_{k} \in \mathbb{S}_{>}^{3}$. By the triality theory, $\boldsymbol{\chi}_{k}(\mathbf{x})$ could be a local minimizer if $\mathbf{T}_{k} \in \mathbb{S}_{<}^{3}$. To see this, let us consider the general case of the equation (75) , i.e., by chain role for $W(\mathbf{F})=V(\mathbf{E}(\mathbf{F}))$, we have

$$
\frac{\partial^{2} W(\mathbf{F})}{\partial F_{\beta}^{i} \partial F_{\beta}^{j}}=\delta^{i j} T_{\alpha \beta}+\sum_{\theta, \nu=1}^{3} F_{\theta}^{i} H_{\theta \alpha \beta \nu} F_{\nu}^{j},
$$

where $\mathbf{H}=\left\{H_{\theta \alpha \beta \nu}\right\}=\nabla^{2} V(\mathbf{E})$. By the convexity of the canonical function $V(\mathbf{E})$, the Hessian $\mathbf{H}$ is positive definite. Therefore, if $\mathbf{T}=\left\{T_{\alpha \beta}\right\} \in \mathbb{S}_{>}^{3}$, the L.H. condition holds. By the fact that $\mathbf{F}=\boldsymbol{\tau} \mathbf{T}^{-1}$, if $\mathbf{T} \in \mathbb{S}_{<}^{3}=\left\{\mathbf{T} \in \mathbb{R}^{3 \times 3} \mid \mathbf{T}=\mathbf{T}^{T}, \mathbf{T} \prec 0\right\}$, the L.H. condition could also hold depending on the eigenvalues of $\mathbf{T}$. Therefore, if the boundaryvalue problem (77) has multiple solutions $\left\{\boldsymbol{\chi}_{k}(\mathbf{x})\right\}$ at $\mathbf{x} \in \Omega$, the operator $A(\boldsymbol{\chi})$ may not be elliptic at $\mathbf{x} \in \Omega$ even if the L.H. condition holds at certain $\chi_{k}(\mathbf{x})$.

For St Venant-Kirchhoff material, the strain energy $V(\mathbf{E})$ is convex (quadratic)

$$
V(\mathbf{E})=\mu \operatorname{tr}\left(\mathbf{E}^{2}\right)+\frac{1}{2} \lambda(\operatorname{tr} \mathbf{E})^{2},
$$

where $\mu, \lambda>0$ are Lamé constants. In this case, the complementary energy is

$$
V^{*}(\mathbf{T})=\frac{1}{4 \mu} \operatorname{tr}\left(\mathbf{T}^{2}\right)-\frac{\lambda}{4 \mu(3 \lambda+2 \mu)}(\operatorname{tr} \mathbf{T})^{2}
$$

and the canonical dual algebraic equation (866) is a cubic symmetrical tensor equation

$$
\mathbf{T}^{2}+\frac{1}{\mu} \mathbf{T}^{3}-\frac{\lambda}{\mu(3 \lambda+2 \mu)}(\operatorname{tr} \mathbf{T}) \mathbf{T}^{2}=\boldsymbol{\tau}^{T} \boldsymbol{\tau}
$$

It was shown in 23 ] that for a given $\boldsymbol{\tau}(\mathbf{x}) \neq 0$, this canonical dual algebraic equation has a unique positive-definite solution $\mathbf{T}_{+} \in \mathbb{S}_{>}^{3}$, at most nine negative-definite solutions 
$\mathbf{T}_{-} \in \mathbb{S}_{<}^{3}$, and at most 17 indefinite solutions at each material point $\mathbf{x} \in \Omega$. By the triality theory, $\mathbf{T}_{+} \in \mathbb{S}_{>}^{3}$ gives the global minimizer of the total potential $\Pi(\chi)$; the smallest $\mathbf{T}_{-} \in \mathbb{S}_{<}^{3}$ leads to local maximizer, while the biggest $\mathbf{T}_{-} \in \mathbb{S}_{<}^{3}$ could give a local minimizer of $\Pi(\chi)$. Detailed discussion is given in [23].

\section{Concluding Remarks and Open Problems}

Concrete applications of the canonical duality-triality theory have been presented in this paper for solving general anti-plane shear problems in finite elasticity. Results show that the nonconvex variational problem could have multiple solutions at each material point $\mathbf{x} \in \Omega$, the Euler equation is not elliptic and the Legendre-Hadamard condition is only a local criterion which can't guarantee uniqueness of solutions. By using the pure complementary energy principle proposed in [15, 16], the nonlinear partial differential equation in finite elasticity is equivalent to an algebraic (tensor) equation, which can be solved, under certain conditions, to obtain a complete set of solutions in stress space. Therefore, an unified analytical solution form is obtained for the nonconvex variational problem. The GaoStrang complementary gap function and the triality theory can be used to identify both global and local extrema. By the fact that the statically admissible stress field $\boldsymbol{\tau} \in \mathcal{T}_{a}$ may not be uniquely determined for a given external force $\mathbf{t}(\mathbf{x})$ on $\Gamma_{t}$, the compatibility condition (42) should be satisfied in order that this analytical solution solves also the mixed boundary-value problem. How to satisfy this compatibility condition and to identify local minimizer for 3-D problems are still open questions and deserve future study.

\section{Acknowledgements}

The topic of this paper was suggested by Professor David Steigmann at UC-Berkeley. Important comments and constructive suggestions from three anonymous referees are sincerely acknowledged. The research was supported by US Air Force Office of Scientific Research (AFOSR FA9550-10-1-0487).

\section{References}

[1] Abeyaratne, R. C. (1980). Discontinuous deformation gradients in plane finite elastostatics of incompressible materials. J. Elasticity 10, 255-293.

[2] Atiyah, M.F. (2007) Duality in Mathematics and Physics, lecture notes from the Institut de Matematica de la Universitat de Barcelona (IMUB).

[3] Ball, J.M. (2002). Some open problems in elasticity. In Geometry, Mechanics, and Dynamics, P. Newton, P. Holmes, and A. Weinstein (Eds), pages 3-59. Springer, New York. 
[4] Barron, E.N. (1999) Viscosity solutions and analysis in $L^{\infty}$, Nonlinear Analysis, Differential Equations and Control, Dordrecht, 1-60.

[5] Cai, K, Gao, D.Y., and Qin, Q.H. (2013). Post-buckling solutions of hyper-elastic beam by canonical dual finite element method, to appear in Mathematics and Mechanics of Solids. Published on line at http://arxiv.org/abs/1302.4136

[6] Ciarlet, P.G. (2013). Linear and Nonlinear Functional Analysis with Applications, SIAM, Philadelphia.

[7] Ciarlet, P.G. (1988). Mathematical Elasticity. Volume I: Three-Dimensional Elasticity. North-Holland.

[8] Dacorogna, B. (1989). Direct Methods in the Calculus of Variations, Springer-Verlag.

[9] Evans, L. C. (2010). Partial differential equations, Graduate Studies in Mathematics 19 (2nd ed.), Providence, RI: American Mathematical Society.

[10] Fosdick, R.L. and Serrin, J. (1973). Rectilinear steady flow of simple fluids. Proceedings of the Royal Society of London. A. Mathematical and Physical Sciences, 332.1590: 311-333.

[11] Gao, D.Y. (1992). Global extremum criteria for nonlinear elasticity, J. Appl. Math. Physics ( ZAMP), 43, pp. 924-937.

[12] Gao, D.Y. (1996). Complementarity and duality in natural sciences, in Philosophical Study in Modern Science and Technology (in Chinese), Tsinghua University Press, Beijing, China, pp. 1225.

[13] Gao, D.Y. (1997). Dual extremum principles in finite deformation theory with applications to post-buckling analysis of extended nonlinear beam theory , Applied Mechanics Reviews, ASME,50, no. 11, Part 2, November 1997, S64-S71.

[14] Gao, D.Y. (1998). Duality, triality and complementary extremum principles in nonconvex parametric variational problems with applications IMAJ. Appl. Math. 61, pp. 199-235.

[15] Gao, D.Y. (1999). Pure complementary energy principle and triality theory in finite elasticity, Mech. Res. Comm. 26 (1), 31-37.

[16] Gao, D.Y. (1999). General analytic solutions and complementary variational principles for large deformation nonsmooth mechanics. Meccanica 34, 169-198.

[17] Gao, D.Y. (2000). Duality Principles in Nonconvex Systems: Theory, Methods and Applications, Kluwer Academic Publishers, Dordrecht /Boston /London, xviii + 454pp.

[18] Gao, D.Y. (2000). Analytic solution and triality theory for nonconvex and nonsmooth variational problems with applications, Nonlinear Analysis, 42, 7, 1161-1193. 
[19] Gao, D.Y. (2000). Canonical dual transformation method and generalized triality theory in nonsmooth global optimization, J. Global Optimization, 17 (1/4), pp. 127160 .

[20] Gao, D.Y. (2003). Perfect duality theory and complete solutions to a class of global optimization problems. Optim. 52(4-5), 467-493.

[21] Gao, D.Y. (2003). Nonconvex semi-linear problems and canonical dual solutions. Advances in Mechanics and Mathematics, Vol. II, D.Y. Gao and R.W. Ogden (ed), Kluwer Academic Publishers, pp. 261-312.

[22] Gao, D.Y. (2004). Complementary variational principle, algorithm, and complete solutions to phase transitions in solids governed by Landau-Ginzburg equation. Mathematics and Mechanics of Solid, 9:285-305.

[23] Gao, DY, Hajilarov, E., and Ogden, RW (2013). On analytic solutions to 3-d finite deformation problems governed by St Venant-Kirchhoff material. Working paper.

[24] Gao, D.Y., Li, J.F. and Viehland, D. (2003). Tri-duality theory in phase transformations of ferroelectric crystals with random defects. Proceedings of IUTAM Symposium on Complementarity, Duality and Symmetry in Nonlinear Mechanics. Kluwer Academic Publishers, pp. 67-84.

[25] Gao, D.Y. and Lv, X.J. (2013). Multiple solutions for non-convex variational boundary value problems in $\mathbb{R}^{n}$, to be submitted.

[26] Gao, D.Y. and Ogden, R.W. (2008). Closed-form solutions, extremality and nonsmoothness criteria in a large deformation elasticity problem, ZAMP, 59:498 - 517.

[27] Gao, D.Y. and Ogden, R.W. (2008). Multiple solutions to non-convex variational problems with implications for phase transitions and numerical computation, Quarterly J. Mech. Appl. Math. 61 (4), 497-522.

[28] Gao, D.Y. and Russell, D.L.(1998). An extended beam theory for smart materials applications: II Static formation problems. Appl. Math. Optim. 38(1), 69-94.

[29] Gao, D.Y. and Sherali, H.D. (2009). Advances in Applied Mathematics and Global Optimization, Springer.

[30] Gao, D.Y. and Sherali, H.D. (2009). Canonical duality: Connection between nonconvex mechanics and global optimization, in Advances in Appl. Mathematics and Global Optimization, D.Y. Gao and H.D. Sherali (Eds), 249-316, Springer.

[31] Gao, D.Y. and Strang, G. (1989). Geometric nonlinearity: Potential energy, complementary energy, and the gap function, Quart. Appl. Math., 47, pp. 487-504. 
[32] Gao, D.Y. and Wu, C. (2012). On the triality theory for a quartic polynomial optimization problem, J. Ind. Manag. Optim. 8(1): 229-242.

[33] Gao, DY, and Wu, C. On the Triality Theory in Global Optimization, to appear in J. Global Optimization http://arxiv.org/abs/1104.2970

[34] Gao, D.Y. and Yang, W.H. (1995). Multi-duality in minimal surface type problems, Studies in Appl. Math., 95: 127-146. MIT.

[35] Gao, D.Y. and Yu, H.F. (2008). Multi-scale modelling and canonical dual finite element method in phase transitions of solids. Int. J. Solids and Structures, 45:3660-3673.

[36] Gurtin, M. and Temam, R. (1981). On the anti-plane shear problem in finite elasticity, J. Elasticity, 11 (2), 197-206.

[37] Hellinger, E. (1914). Die allgemeine Ansätze der Mechanik der Kontinua. Encyklopädie der Mathematischen Wissenschaften IV, 4, 602-94.

[38] Hill, JM (2001) A review of partial solutions of finite elasticity and their applications, Int. J.of Nonlinear Mechanics, 36, 447-463.

[39] Hill, JM and Milan, AM (2001) Finite elastic plane strain bending of sectors of circular cylindrical sectors, Int J. Engineering Science, 39, 209-227.

[40] Holzapfel, G.A. (2000). Nonlinear Solid Mechanics: A Continuum Approach for Engineering. Wiley. ISBN 978-0471823193.

[41] Holzapfel, G.A. and Ogden, R.W., (Eds.) (2009). Biomechanical Modelling at the Molecular, Cellular and Tissue Levels. Series: CISM courses and lectures. Springer, Vienna, Austria.

[42] Horgan, C.O. (1995). Anti-Plane Shear Deformations in Linear and Nonlinear Solid Mechanics, SIAM Review, 37(1), 53-81.

[43] Horgan, C.O. and Saccomandi, G. (2001). Antiplane shear deformations for nonGaussian isotropic, incompressible hyperelastic materials. Proc. Roy. Soc. London A 457, 1999-2017.

[44] Jiang, Q. and Knowles, J.K. (1991). A class of compressible elastic materials capable of sustaining finite anti-plane shear, J. Elasticity, Volume 25 (3), pp. 193-201

[45] Knowles, J.K. (1976). On finite anti-plane shear for incompressible elastic materials, J. Australia Math. Soc., 19, 400-415.

[46] Knowles, J.K. (1977). The finite anti-plane shear field near the tip of a crack for a class of incompressible elastic solids, Internat. J. Fracture, 13, pp. 611-639. 
[47] Koiter, W.T. (1976). On the complementary energy theorem in nonlinear elasticity theory, Trends in Appl. of Pure Math. to Mech. ed. G. Fichera, Pitman.

[48] Lee, S. J. and Shield, R. T. (1980). Variational principles in finite elasticity, J. Appl. Math. Physics (ZAMP), 31, 437-453.

[49] Lee, S. J. and Shield, R. T. (1980). Applications of variational principles in finite elasticity, J. Appl. Math. Physics (ZAMP), 31, pp 454-472.

[50] Levinson, M. (1965). The complementary energy theorem in finite elasticity, Trans. ASME, ser. E, J. Appl. Mech., 87, pp. 826-828.

[51] Li, S.F. and Gupta, A. (2006). On dual configuration forces, J. of Elasticity, 84:13-31.

[52] Luo, J. and Wang, X. (2009). On the anti-plane shear of an elliptic nano inhomogeneity European Journal of Mechanics A/Solids, 28: 926 - 934.

[53] Marsden, J.E. and Hughes, T.J.R. (1983). Mathematical Foundations of Elasticity, Prentice-Hall.

[54] Morrey, C.B. (1966). Multiple Integrals in the Calculus of Variations, Springer, Berlin.

[55] Murty, KG and Kabadi, SN (1987). Some NP-complete problems in quadratic and nonlinear programming. Math. Programming, 39, 117 - 129.

[56] Narita, F. and Shindo, Y. (1998). Layered piezoelectric medium with interface crack under anti-plane shear Theoretical and Applied Fracture Mechancis, 30(2), 119-126.

[57] Ogden, R.W. (1975). A note on variational theorems in non-linear elastostatics. Math. Proc. Camb. Phil. Sco., 77, 609-615.

[58] Ogden, R.W. (1977). Inequalities associated with the inversion of elastic stressdeformation relations and their implications, Math. Proc. Camb. Phil. Soc., 81, 313324 .

[59] Ogden, RW (1984/97). Non-Linear Elastic Deformations, Ellis Horwood/Dover.

[60] Oden, J.T. and Reddy, J.N. (1983). Variational Methods in Theoretical Mechanics. Springer-Verlag.

[61] Paulino, G. H. Saif, M.T.A. Mukherjee, S. (1993). A finite elastic body with a curved crack loaded in anti-plane shear International Journal of Solids and Structures, 30 (8), 10151037

[62] Pucci, E., Rajagopal, K.R. , Saccomandi, G (2014). On the determination of semiinverse solutions of nonlinear Cauchy elasticity: The not so simple case of anti-plane shear. Int. J. of Engineering Science, http://dx.doi.org/10.1016/j.ijengsci.2014.02.033 
[63] Pucci E., Saccomandi G. (2013). The anti-plane shear problem in non-linear elasticity. Journal of Elasticity, 113, 167-177.

[64] Ressner, E. (1953). On a variational theorem for finite elastic deformations, J. Math. Phys., 32(2-3), 129-135.

[65] Sewell, M.J. (1987). Maximum and minimum principles: A unified approach, with applications, Cambridge University Press, 468pp.

[66] Shubin, M. A. (2001). Elliptic operator, in Encyclopedia of Mathematics, M. Hazewinkel (eds), Springer.

[67] Silling SA (1988). Consequences of the Maxwell relation for anti-plane shear deformations of an elastic solid Journal of elasticity, 19 (3), pp 241-284

[68] Strugariu, R., Voisei, M.D., and Zalinescu, C.(2011). Counter-examples in bi-duality, triality and tri-duality, Discrete and Continuous Dynamical Systems - Series A (DCDS-A) 31, 1453-1468.

[69] Veubeke, B.F. (1972). A new variational principle for finite elastic displacements. Int. J. Engineering Science, 10, pp. 745-763.

[70] Voisei, M.D., Zalinescu, C. (2012). Some remarks concerning Gao-Strang's complementary gap function, Applicable Analysis, DOI: 10.1080/00036811.2010.483427.

[71] Yu, H.H. and Yang, Wei (1994). Mechanics of transonic debonding of a bimaterial interface: The anti-plane shear case Journal of the Mechanics and Physics of Solids, Volume 42(11): 1789 - 1802 
(a)

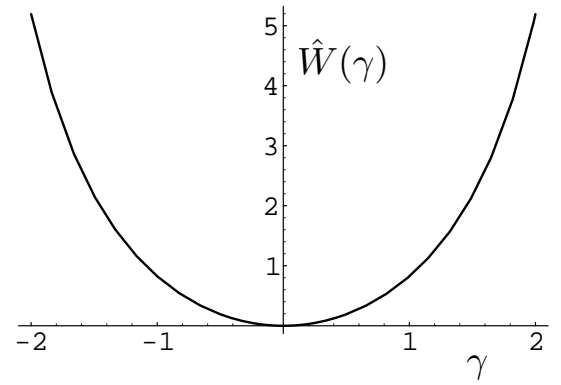

(b)

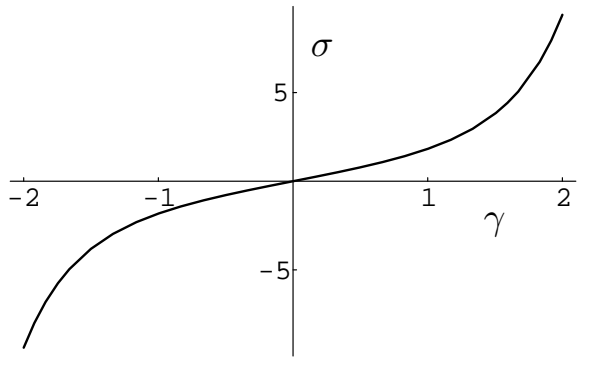

